\title{
Sub-linear integration underlies binocular processing in primary visual cortex
}

\section{Fabio Longordo ${ }^{1}$, Minh-Son To ${ }^{1,2}$, Kaori Ikeda ${ }^{1}$ and Greg J. Stuart ${ }^{1}$}

1Eccles Institute of Neuroscience, John Curtin School of Medical Research, Australian National University, Canberra, Australia

2Department of Human Physiology and Centre for Neuroscience, Flinders University Adelaide, Australia

Address correspondence to: Fabio.Longordo@anu.edu.au \& Greg.Stuart@anu.edu.au

Key Words: cortex, pyramidal neuron, dendrite, summation, vision, mouse 


\begin{abstract}
While we know much about the capacity of neurons to integrate synaptic inputs in vitro, less is known about synaptic integration in vivo. Here we address this issue by investigating the integration of inputs from the two eyes in mouse primary visual cortex. We find that binocular inputs to layer $2 / 3$ pyramidal neurons are integrated sub-linearly in an amplitude-dependent manner. Sub-linear summation was greatest when binocular responses were largest, as occurs at the preferred orientation and binocular disparity, and highest contrast. Based on voltage-clamp experiments and modeling, we show that sub-linear summation occurs postsynaptically. The extent of sub-linearity cannot be accounted for solely by non-linear integration of excitatory inputs, even when they are activated closely in space and time, but requires balanced recruitment of inhibition. Finally, we show that sub-linear binocular summation acts as a divisive form of gain control, linearizing the output of binocular neurons and enhancing orientation selectivity.
\end{abstract}




\section{Introduction}

Neurons in the brain typically receive thousands of synaptic inputs, which are integrated in time and space to generate an output signal. As the vast majority of these inputs are made on the dendritic tree, research over many years has focused on understanding how the passive and active properties of dendrites influence synaptic integration. A range of forms of synaptic integration have been described, from sub-linear, as seen in passive dendrites ${ }^{1}$, to supralinear due to generation of dendritic spikes ${ }^{2-8}$. More subtle modifications of synaptic integration have also been described due to activation of potassium, HCN and persistent sodium channels ${ }^{9-12}$. Evidence for these different forms of synaptic integration is based largely on experiments conducted in vitro. Much less is known about how neurons process synaptic inputs while embedded in their network in vivo.

The cortex provides an ideal brain region where this issue can be addressed. Single neurons in sensory cortical areas integrate inputs with defined spatial and temporal patterns depending on the characteristics of the stimulus. These early computations are thought to be crucial to the processing of sensory information. While there is emerging evidence that somatosensory stimulation can evoke active forms of dendritic integration in vivo ${ }^{13-16}$, to what extent this can be generalized across different sensory modalities is unclear. In vitro work indicates that supra-linear forms of synaptic integration require correlated activity clustered onto the same dendritic location or branch ${ }^{3,8}$. In contrast, when inputs are dispersed onto different branches, or activated at different times, linear or sub-linear forms of synaptic integration are usually seen ${ }^{8,17-19}$. It is only now becoming apparent how synaptic inputs, encoding specific sensory information, are distributed at the dendritic level. Some evidence indicates that sensory information is processed by dendrites in a dispersed manner ${ }^{20,21}$, which is less likely to recruit 
active dendritic processing, whereas other evidence supports clustered activation of synaptic inputs onto the same dendrite 22,23 .

Here we examine the integration of synaptic inputs in the binocular region of the primary visual cortex of the mouse in vivo. By definition, binocular neurons code information from the two eyes, providing a model system in which to study how two defined sensory inputs are integrated at the single cell level. While extracellular recording has provided a basic understanding of how simple and complex cells integrate binocular information ${ }^{24,25}$, this analysis is based exclusively on the firing output of neurons, and therefore lacks information on the integration of the underlying synaptic responses. Here we used whole-cell patch-clamp recording in vivo to study the synaptic events leading to binocular processing in layer $2 / 3$ pyramidal neurons. We find that small synaptic inputs from the two eyes are integrated linearly, whereas large inputs are integrated sub-linearly. Using voltage-clamp and compartmental modeling we show that sub-linear summation cannot be explained solely by non-linear integration of excitatory inputs, but requires balanced recruitment of inhibition. Finally, we find that sub-linear summation of binocular synaptic inputs acts as a divisive form of gain control, linearizing the output of binocular neurons and enhancing orientation selectivity. 


\section{Results}

To investigate the integration of binocular inputs we made in vivo whole-cell current-clamp recordings from layer $2 / 3$ pyramidal neurons in primary visual cortex of anaesthetized adult mice. Visual stimuli (sinusoidal drifting gratings) were presented selectively to each eye alone, or both eyes together, by using computer-controlled motorized eye shutters (Fig. 1a). To reveal the underlying synaptic response action potentials were removed using a sliding median filter ${ }^{26}$ (Fig. 1a; bottom). This approach did not significantly affect the measures we used to characterize subthreshold synaptic responses (Supplementary Fig. 1). Medianfiltered voltage responses were averaged across trials, as well as over a single cycle of the visual stimulus, fitted with a sinusoidal function and the peak $\left(\mathrm{V}_{\text {peak }}\right)$, mean $\left(\mathrm{V}_{\text {mean }}\right)$, and amplitude of sinusoidal modulation ( $\left.\mathrm{V}_{\text {modulation }}\right)$ were quantified (Fig. 1b).

\section{Binocular synaptic inputs sum sub-linearly}

We first restricted our analysis to neurons classified as simple cells (Supplementary Fig. 2a,b) and focused on synaptic potentials evoked by stimuli at the preferred orientation. That is, the orientation that gave the largest suprathreshold (spiking) response during stimulation of both eyes together. Consistent with earlier work, in adult mice the preferred orientation during stimulation of the ipsilateral or contralateral eye alone was matched in the majority of cells $^{27}$, and was similar to that during stimulation of both eyes together (Supplementary Fig. 2c). To investigate the integration of synaptic inputs from the two eyes we compared the synaptic response evoked by stimulation of both eyes together to the linear sum of responses evoked by stimulation of each eye separately. Since neurons were poorly direction tuned, with $\sim 85 \%$ of cells having a direction selectivity index less than $0.5^{28}$ (Supplementary Fig. 2e), responses to both directions were pooled. We found that inputs from the contralateral 
and ipsilateral eye added linearly in cells where the expected linear sum was small, however, sub-linear summation was observed in cells where the expected linear sum was large (Fig. 1c,d). Overall, at the preferred orientation, sub-linear summation of binocular synaptic inputs was observed when the peak of the expected linear sum was larger than approximately $15 \mathrm{mV}$ (Fig. 1e), with the extent of sub-linear summation proportional to the amplitude of the expected linear sum (Fig. 1f). Sub-linear summation did not depend on the stimulus direction (Supplementary Fig. 3a,b). Similar observations were made in neurons classified as complex cells (Supplementary Fig. 4a-c).

We next investigated whether the summation of synaptic potentials depended on stimulus orientation (Fig. 2a). When averaging across cells significant sub-linearity was only observed at or near the preferred orientation (Fig. 2b). This may arise because responses at the preferred orientation are by definition largest, or because the cellular mechanisms generating sub-linear summation depend on stimulus orientation. To investigate this we tested how binocular summation depends on the amplitude of the expected linear sum during gratings with different orientations. This analysis showed that in individual cells inputs from the two eyes could summate sub-linearly at non-preferred orientations if the expected linear sum was large (Fig. 2c,d). These data indicate that the orientation dependence of sub-linear summation (Fig. 2b) arises simply because the proportion of responses with large expected linear sums $(>15 \mathrm{mV})$ is highest at the preferred orientation (Fig. 2d; numbers in brackets, p $<0.05, \chi^{2}$ test).

Selective presentation of the same visual stimulus to the contralateral or the ipsilateral eye alone evoked responses that were often out of phase (Fig. 2e). Notably, the extent of sublinear summation was greatest when the phase difference between monocular responses was 
smallest (Fig. 2e,f). As seen for stimulus orientation, this phase dependence of binocular integration was due to the higher proportion of responses with large expected linear sums $(>15 \mathrm{mV}$ ) when monocular responses were in phase (Fig. 2g,h; numbers in brackets, p < 0.01, $\chi^{2}$ test). Finally, we investigated the contrast sensitivity of binocular integration. Stimuli were presented at three different contrasts (30, 50 and $100 \%$; Supplementary Fig. 3c). The peak of the synaptic response to stimulation of the contralateral or ipsilateral eye alone increased with increasing contrast (Supplementary Fig. 3d), leading to larger expected linear sums at high contrast. Consistent with an amplitude-dependent effect, the extent of sub-linear summation of binocular responses was greatest at highest contrast (Supplementary Fig. $3 \mathbf{e}, \mathbf{f})$.

One of the major roles of binocular integration is to encode binocular disparity, which is thought to be critical for depth perception. To study how binocular summation depends on the stimulus phase disparity we made recordings from binocular neurons during presentation of binocular stimuli at six different inter-ocular spatial phase disparities at the preferred orientation (Fig. 3a). In these experiments, the initial phase of gratings presented to one eye was fixed, while the initial phase of the stimulus to the other eye was systematically varied in 60 degrees increments (Fig. 3b). First, we presented the stimuli selectively to each eye alone. Changes in the initial phase of the stimulus to one eye led to almost identical changes in the phase of the corresponding monocular synaptic response (Fig. 3b,c). We then presented the stimuli to both eyes together and studied how synaptic responses summate as a function of the inter-ocular phase disparity (Fig. 3d). At the neuron's preferred phase disparity, defined as the phase disparity that gave the largest suprathreshold (spiking) response (Fig. 3e,f, top), the phase difference between monocular responses was smallest (Fig. 3e,f, bottom), and the extent of sub-linearity was greatest (Fig 3g). This observation, at the single cell level, is 
essentially identical to that observed across the cell population when phase differences between monocular responses were observed during presentation of the same visual stimulus to both eyes (see Fig. 2f). In summary, these data show that the extent of sub-linear summation is largest at the neuron's preferred phase disparity, when monocular responses are in phase leading to binocular responses of largest amplitude.

Membrane potential responses of simple cells to drifting gratings are commonly characterized by the mean voltage change during the stimulus and by the amplitude of sinusoidal modulation (Fig. 1b). Previous work suggests that the cellular mechanisms underlying these components may be different, and may code different aspects of the stimulus ${ }^{29}$. We therefore investigated the integration of these different components during binocular stimulation (Fig. 4a). The amplitude of both the mean and modulation components of the binocular response displayed weak orientation tuning (Fig. 4b,c), with sinusoidal modulation having a higher orientation selectivity index (Fig. 4d). To investigate how these different components of the synaptic response summate during binocular stimulation, we compared the linear sum of each component during stimulation of each eye on its own to the response observed during stimulation of both eyes together (Fig. 4e,f). While there was significant scatter around a line with slope of one (indicating linear summation), when the data were sorted based on peak amplitude we observed sub-linear summation of both modulation and mean components when the expected linear sum was large (Fig. $\mathbf{4 g}$ ).

Taken together, these results indicate that sub-linear binocular integration is not triggered by a subset of incoming synaptic inputs coding a specific aspect of the stimulus (e.g. preferred versus non preferred phase disparity). Furthermore, sub-linear summation is not restricted to a specific component of the response (mean or modulation). Instead, the extent of sub-linear 
binocular integration depends exclusively on the amplitude of the responses evoked by stimulation of each eye on its own. We therefore conducted a point-by-point analysis of summation of binocular synaptic inputs in individual cells (Fig. $\mathbf{4 h}$; in $10 \mathrm{~ms}$ epochs). This analysis revealed a strong correlation between the extent of sub-linear summation and the amplitude of the expected linear sum during a single visual stimulus (Fig. 4i), with an average correlation coefficient of $-0.51 \pm 0.06$ across all cells at the preferred orientation ( 40 responses, $\mathrm{n}=20$ cells; $\mathrm{p}<0.05$ ). Applying this point-by-point analysis across all simple cells at all 12 directions produced a stimulus independent description of the dependence of sub-linear summation on the amplitude of the expected linear sum of contralateral and ipsilateral inputs (Fig. 4j). Similar observations were made in complex cells (Supplementary Fig. 4d,e).

\section{Mechanisms underlying sub-linear binocular integration}

The observed sub-linear summation of binocular synaptic potentials may have a presynaptic origin, due to a reduction in excitation or an increase in inhibition during large responses, or could occur postsynaptically due to non-linear interactions within layer 2/3 pyramidal neurons. To address these possibilities we investigated how excitatory and inhibitory postsynaptic currents (EPSCs and IPSCs) summate during binocular stimulation using somatic whole-cell voltage-clamp. Recordings were made at hyperpolarized (-80 mV; $\mathrm{n}=12$ cells) and depolarized $(+20$ to $+30 \mathrm{mV}$; $\mathrm{n}=10$ cells) potentials to isolate the excitatory and inhibitory components of the binocular response, respectively ${ }^{30,31}$. EPSCs recorded at the predicted reversal potential for inhibition $(-80 \mathrm{mV})$ during stimulation of both eyes were orientation tuned (Fig. 5a,b), consistent with previous data in mice during monocular stimulation ${ }^{30,31 .}$ Importantly, EPSCs evoked by stimulation of both eyes were similar to the linear sum of

EPSCs evoked during stimulation of each eye alone (Fig. 5c). Similarly, during stimulation of both eyes together, IPSCs recorded at depolarized potentials were well predicted by the linear 
sum of IPSCs evoked during stimulation of each eye alone (Fig. 5d). These data indicate that inhibitory and excitatory conductance changes sum essentially linearly, arguing against the idea that sub-linear summation of voltage response during binocular stimulation results from reduced excitation or increased inhibition.

We next studied the relationship between excitation and inhibition in single cells during binocular stimulation at the preferred orientation. Excitatory $\left(\mathrm{g}_{\mathrm{e}}\right)$ and inhibitory $\left(\mathrm{g}_{\mathrm{i}}\right)$ conductances were estimated using previously published methods ${ }^{32,33}$. This analysis revealed that $g_{e}$ and $g_{i}$ in individual neurons increased in a proportional manner during stimulation of both eyes together (Fig. 5e; average correlation $=0.95 \pm 0.01, \mathrm{n}=9$ cells; $\mathrm{p}<0.01$ ). The ratio of inhibition to excitation $\left(\mathrm{g}_{\mathrm{i}} / \mathrm{g}_{\mathrm{e}}\right)$ was estimated from the slope of linear fits to data from individual cells and indicated that inhibition and excitation were recruited in a balanced manner with an average ratio close to one $(1.12 \pm 0.18 ; n=9)$. Consistent with this analysis, the average reversal potential of evoked synaptic currents during binocular stimulation was approximately halfway between the predicted reversal potential for excitation and inhibition (average: $-45.8 \pm 3.7 \mathrm{mV} ; \mathrm{n}=9$ ). These data indicate that excitation and inhibition are recruited in a balanced manner during binocular visual input.

That EPSCs and IPSCs sum essentially linearly during binocular stimulation suggests that sublinear summation of synaptic potentials has a postsynaptic origin. To investigate this further we simulated visual responses in a morphologically realistic model of a layer $2 / 3$ pyramidal neuron where contralateral and ipsilateral excitatory and inhibitory inputs were recruited linearly in a balanced manner in order to match our experimental observations (Fig. 5e). We modeled two scenarios. In one scenario contralateral and ipsilateral inputs converge upstream from layer 2/3, for example in layer 4 (Fig. 6a, left), in the second scenario separate 
contralateral and ipsilateral inputs converge onto layer 2/3, either from layer 4 neurons with different ocular dominance (Fig. 6a, middle) ${ }^{34}$ or via different thalamic or hemispheric pathways (Fig. 6a, right) ${ }^{35}$. To simulate these two scenarios ipsilateral and contralateral excitatory inputs were sampled either from a common pool or from two segregated pools of synapses distributed randomly onto basal dendrites (Fig. 6b, top). Inhibitory inputs were distributed randomly onto basal dendrites or placed at the soma (Fig. 6b, bottom). We simulated our voltage-clamp data (Fig. 5) using a realistic value of the somatic series resistance (35 $\mathrm{M} \Omega$ ) and adjusted the resting membrane properties of the model to match the average holding currents recorded at hyperpolarized and depolarized potentials. Excitatory and inhibitory inputs were randomly activated via sinusoidally modulated independent Poisson processes in a balanced manner so that the magnitude and ratio of inhibitory to excitatory synaptic conductances estimated from somatic voltage-clamp simulations was similar to that observed experimentally (Supplementary Fig. 5; compare with Fig. 5e). As one would expect due to space-clamp errors associated with voltage-clamping neurons with complex dendritic trees ${ }^{36}$, the real excitatory and inhibitory conductances required to match the experimental data were significantly larger than that estimated from somatic voltageclamp, particularly in models with dendritic inhibition (Supplementary Fig. 5). Furthermore, proportionally more inhibition was required to simulate the experimentally recorded inhibitory to excitatory conductance ratio $\left(g_{i} / g_{e}=1.12\right)$ in models with dendritic inhibition, whereas the opposite was the case in models with somatic inhibition (Supplementary Fig. 5). These simulations highlight issues with the interpretation of excitatory and inhibitory conductance estimates from somatic voltage-clamp in neurons with dendrites.

Linear recruitment of ipsilateral and contralateral excitatory inputs separately or together in a balanced manner with inhibition generated excitatory and inhibitory currents that summed 
in a manner similar to that observed experimentally (Fig. 6c). Essentially no difference in voltage-clamp responses at the soma was observed in models with common compared to segregated ipsilateral and contralateral excitatory inputs (Fig. 6c). These simulations predicted a small amount of sub-linear summation of excitatory and inhibitory currents during large responses, which was absent in models with current-based synapses (Fig. 6d), indicating that it results from poor voltage- or space-clamp. The capacity of this linear recruitment model to accurately predict our voltage-clamp data further substantiates our conclusion that sub-linear summation of voltage responses arises postsynaptically, and is not due to a decrease in excitatory drive or an increase in inhibitory drive during large binocular responses.

We next used these models to simulate voltage responses at the soma during stimulation of contralateral and ipsilateral inputs alone or together. In these simulations the passive properties of the model were adjusted to match those observed experimentally at the resting membrane potential during current-clamp recordings. These simulations accurately predicted the extent of sub-linear summation of voltage responses observed experimentally during binocular integration (Fig. 6e,f). Importantly, we were not able to distinguish between models with common or segregated ipsilateral and contralateral input, indicating either model is valid. Furthermore, we could not distinguish between models with somatic or dendritic inhibition.

As the extent of sub-linear summation depends on the relative location of synaptic inputs we generated additional models with different spatial distributions of excitatory input; either dispersed randomly through the entire basal dendritic tree, partitioned into different basal dendritic regions, or concentrated onto a single dendritic branch (Fig. 7a). Inhibition was not 
included in these models to determine the extent with which non-linear interactions between ipsilateral and contralateral excitatory inputs alone contribute to sub-linear summation of binocular inputs. As observed experimentally, small contralateral and ipsilateral responses summated linearly, whereas larger responses summated sub-linearly (Fig. $7 \mathbf{b}, \mathbf{c}$ ). The extent of sub-linear binocular summation was greatest when contralateral and ipsilateral synapses were concentrated onto a single dendritic branch, however, even in this extreme case sublinear summation was significantly less than that observed experimentally (Fig. 7c).

Finally, we tested the impact of temporal correlations between excitatory inputs. Temporal correlations were made either within (monocular correlation) or between (binocular correlation) contralateral and ipsilateral inputs by using the same Poisson input train to drive multiple sets of synapses in the dispersed model (Fig. 7d). We characterized the extent of these correlations by comparing the number of activated excitatory inputs within different time windows (Fig. 7e). As expected, the introduction of temporal correlations increased the proportion of synapses that were simultaneously activated, however, the difference between uncorrelated and correlated models rapidly decayed when we considered correlations over larger time windows, still relevant for non-linear interaction (Fig. 7e,f). As a result, introduction of temporal correlations either within or between contralateral and ipsilateral inputs did not significantly affect the degree of sub-linear summation (Fig. 7g). Together, these simulations reveal that non-linear interactions between excitatory inputs alone is not sufficient to explain the extent of sub-linear binocular integration observed experimentally, emphasizing the importance of balanced recruitment of inhibition.

Impact of sub-linear summation on action potential output 
What is the impact of sub-linear summation on action potential output? To address this issue we first described the relationship between the underlying membrane potential and action potential output in individual cells with a previously described model (Fig. 8a) ${ }^{19}$. Using this model we could accurately predict the firing rate observed experimentally based on medianfiltered voltage responses in single cells, confirming its validity (Fig. 8b). We then used this model to predict action potential output assuming linear summation of synaptic responses, and compared the predicted firing rate to that observed experimentally (Fig. 8c,d). As expected from the highly non-linear relationship between membrane potential and action potential output (Fig. 8a), firing rates observed during stimulation of both eyes together were significantly lower than that predicted by the linear sum of synaptic potentials during stimulation of each eye separately (Fig. 8d). Furthermore, when the observed and predicted firing rates were plotted against the linear sum of monocular responses, these data indicate that sub-linear summation significantly reduces the gain of the binocular input/output relationship (Fig. 8e). Importantly, this reduction in gain acts to "linearize" the output of binocular cells, such that the firing rate during binocular stimulation is equal to the linear sum of the firing rates during stimulation of each eye on its own (Fig. 8f; linear fit to the observed spike rates: slope $\left.=1.02 \pm 0.06, \mathrm{r}^{2}=0.6, \mathrm{p}<0.001\right)$. A similar impact of sub-linear summation on the gain of the binocular input/output relationship was observed in complex cells (Supplementary Fig. 4f).

We next characterized the impact of sub-linear summation on the tuning properties of individual neurons during drifting gratings. Consistent with a divisive transformation of the input/output relationship, firing rates were reduced significantly more at the preferred compared to the orthogonal orientation (Fig. 8g; orthogonal reduction=3.47 \pm 0.97 spikes/sec, preferred reduction $=6.81 \pm 1.95$ spikes $/ \mathrm{sec} ; \mathrm{n}=20 ; \mathrm{p}<0.05$, paired t-test). Analysis of tuning 
properties based on observed and predicted firing rates showed that on average binocular integration of synaptic potentials did not alter the direction selectivity index (Fig. 8h) or tuning half width (Fig. 8i), but enhanced the orientation selectivity index (Fig. 8j). A similar impact of sub-linear integration on the orientation selectivity index was observed in a subset of cells with "in-phase" monocular responses at the preferred orientation (Supplementary Fig. 6). This additional analysis indicates that the impact of sub-linear binocular integration on orientation selectivity is not a result of a complex interaction between stimulus orientation and the relative phase of contralateral and ipsilateral responses. 


\section{Discussion}

Here we describe the integration of binocular synaptic inputs in layer $2 / 3$ pyramidal neurons from mouse primary visual cortex. The main finding is that small inputs from the two eyes are integrated linearly, whereas large inputs are integrated sub-linearly. Sub-linear summation depends solely on the amplitude of the incoming inputs and is not restricted to information coding particular aspects of the visual stimulus such as orientation, direction, contrast or binocular phase disparity. The observed sub-linearity is not a result of network interactions up-stream from layer $2 / 3$ pyramidal neurons, but occurs postsynaptically due to non-linear interactions between excitatory inputs together with balanced recruitment of inhibition. This amplitude-dependent sub-linear integration reduces the gain of the input/output relationship of binocular neurons, which linearizes action potential output and increases orientation selectivity.

Previous work in vitro has indicated that neurons can integrate inputs in sub-linear, linear and supra-linear regimes, 2,3,7,8,17,18,37-39. Synaptic inputs in these studies were activated using non-physiological stimuli under artificial conditions. How the different types of integration observed in these in vitro studies relate to synaptic integration in vivo during encoding of physiologically relevant information is unclear. Integration of binocular visual input in the visual cortex provides an ideal system for studying this issue. In mice, as in higher mammals including humans, visual inputs from the two eyes terminate in distinct and well-defined areas of the thalamus before passing onto primary visual cortex where they converge onto single neurons ${ }^{40-42}$. By presenting the two eyes with independently controlled visual stimuli it is possible to see how distinct aspects of visual information are integrated at the single cell level. Under our experimental conditions, we find that binocular inputs are integrated either 
linearly or sub-linearly, but not supra-linearly, suggesting that active dendritic mechanisms are not recruited during binocular integration. Notably, sub-linear summation during binocular integration depended exclusively on the amplitude of the incoming binocular inputs, and was not related to peculiar aspects of the visual information. It therefore represents a general mode of integration in these neurons that is likely to occur under a range of stimulus conditions. The linear and sub-linear modes of dendritic integration that we observed are consistent with recent data showing that integration of visual information in layer 2/3 pyramidal neurons occurs via summation of distributed rather than clustered inputs $^{43}$. In contrast, evidence exists for supra-linear dendritic integration of somatosensory information in the dendrites of cortical layer 5 pyramidal ${ }^{13-15}$ and layer 4 spiny stellate neurons ${ }^{16}$.

Although it is well documented that the convergence of the inputs from the two eyes first happens at the level of the cortex ${ }^{40-42}$, whether this convergence happens at the level of layer $2 / 3$ pyramidal neurons is not known. Preprocessing of binocular input upstream of layer $2 / 3$ pyramidal neurons could in principle explain the observed sub-linear summation. This could occur via a decrease in excitatory input from layer 4 during strong binocular input, due to increased inhibition within layer 4. Alternatively, there could be an increase in inhibitory input to layer 2/3 neurons during large binocular responses, due to enhanced recruitment of feed-forward inhibition. Our voltage-clamp data argue against these possibilities by showing that during binocular integration both excitatory and inhibitory currents sum linearly (Fig. 5c,d). Furthermore, our voltage clamp data were well described by models using linear recruitment of excitatory and inhibitory input (Fig. 6c). The capacity of this "postsynaptic" model to accurately reproduce the extent of sub-linear summation observed experimentally during binocular input (Fig. 6e,f) strengthens the conclusion that sub-linear summation is 
due to non-linear interactions within layer $2 / 3$ pyramidal neurons during balanced recruitment of excitatory and inhibitory binocular inputs. Notably, this mechanism depends exclusively on the number of activated inputs but not on their origin. Indeed, our simulations show that the extent of sub-linear summation observed experimentally was independent of whether binocular inputs were integrated up-stream of layer $2 / 3$ neurons (common pool model) or arise via segregated ipsilateral and contralateral inputs (segregated model).

During binocular stimulation we found that inhibition is recruited in a proportional manner with excitation, with an inhibitory to excitatory conductance ratio close to one. Models with only excitatory synapses could not reproduce the extent of sub-linear integration observed experimentally, even when we pushed these models using extreme scenarios with all excitatory inputs concentrated on the same branch or activated with high instantaneous temporal correlations. We conclude, therefore, that the recruitment of balanced inhibition is essential for the observed sub-linear summation. Addition of inhibition makes the net reversal potential of the binocular responses more hyperpolarized than with excitation alone. This increases the impact of changes in membrane potential on the driving force for current flow, and thereby enhances sub-linear integration. Together with other data 44,45 , these findings provide further evidence that balanced recruitment of excitation and inhibition is critical for sensory processing. Moreover, these results show that postsynaptic sub-linear summation is a robust mechanism that, in comparison to supra-linear dendritic computation, is not very dependent on the precise location of incoming inputs within the dendritic tree.

One of the major computations thought to be performed by binocular neurons is the detection of binocular disparity, which presumably contributes to depth perception of the outside world ${ }^{46,47}$. Our data contributes to an understanding of how inter-ocular phase differences are 
integrated at the single cell level. Numerous single-unit (extracellular) studies in cats have explored the way binocular neurons combine monocular inputs to encode binocular disparity24,25,46-48. These studies have proposed that, for the large majority of cortical cells, integration of binocular inputs can be explained by linear summation of the neuronal signals received from each eye, and is strongly dependent on the phase disparity of gratings presented to the eyes. As these studies are based on action potential output they did not provide direct information on how sub-threshold synaptic inputs are integrated. By recording intracellular voltage changes from layer $2 / 3$ pyramidal neurons during presentation of stimuli with different binocular phase disparities, we show that at the preferred phase disparity the underlying synaptic responses are essentially in phase and are integrated sublinearly. Importantly, this sub-linear summation of synaptic potentials leads to linear summation of monocular firing rates during binocular stimulation (Fig. 8f), consistent with the linearity of binocular integration observed in earlier studies using extracellular recording $24,25,48$.

At the functional level, we find that sub-linear integration of binocular inputs leads to a reduction in gain of the input/output relationship. This divisive transformation has a powerful suppressive effect on the firing rate, compressing the dynamic range of neuronal output and yet does not negatively affect the tuning properties of binocular neurons. Indeed, compared to what would happen if monocular synaptic potentials summed linearly, neurons maintain their ability to discriminate between stimulus contrast and inter-ocular phase disparities and show enhanced orientation selectivity (Fig. 8i). This latter result can be explained by considering the impact of sub-linear integration on the firing output at nonpreferred orientations ${ }^{49}$. Sub-linear summation of synaptic potentials often caused the response to stimulation of both eyes at non-preferred orientations to be below action 
potential threshold, reducing the average firing rate at non-preferred orientations to baseline noise levels. This effect on firing rate at non-preferred orientations can explain the observed increase in the orientation selectivity index during binocular integration.

Finally, one of the key observations in our study is the important role of inhibition in sublinear integration of binocular inputs. Recent findings in mouse primary visual cortex have suggested that distinct subclasses of cortical interneurons, targeting specific neuronal compartments, are responsible for mediating different transformations of the input/output relationship of pyramidal neurons ${ }^{31,49,50}$. While our simulations do not allow us to identify the location of inhibition recruited during binocular integration, the observed impact of binocular sub-linear summation on orientation tuning is very similar to that found during optogenetic activation of parvalbumin-expressing interneurons ${ }^{31}$. This may suggest that inhibition recruited during binocular integration is largely somatic in origin. Future studies will be required to resolve the specific interneuron sub-types recruited during binocular integration, and the role these neurons play in depth perception and stereopsis. 


\section{Methods}

All the procedures were conducted in accordance with the guidelines approved by the Animal Ethics Committee of the Australian National University.

\section{In vivo physiology}

Adult post-critical period male and female C57BL/6 mice (8-10 weeks of age) were anaesthetized with urethane $(0.5-1 \mathrm{~g} / \mathrm{kg}, 10 \% \mathrm{w} / \mathrm{v}$ in saline, i.p.) supplemented with the sedative chlorprothixene $(5 \mathrm{mg} / \mathrm{kg}, 10 \% \mathrm{w} / \mathrm{v}$ in saline, i.p.). In some cases animals were initially anesthetized with ketamine/xylazine $(20 \mathrm{mg} / \mathrm{kg}$ and $3 \mathrm{mg} / \mathrm{kg}$, i.p. $)$ plus chlorprothixene (5mg/kg, i.p.) and maintained with isoflurane (0.5 to $1 \%$ ). All the cells recorded under isoflurane anesthesia and included in the analysis were classified as simple cells $(n=7)$. As results obtained in these experiments were similar to those observed under urethane anesthesia the data have been pooled. Atropine $(0.3 \mathrm{mg} / \mathrm{kg}, 10 \% \mathrm{w} / \mathrm{v}$ in saline) was administered subcutaneously to reduce secretions. The level of anaesthesia was assessed by loss of withdrawal reflexes in response to paw pinch. Rectal temperature was monitored and maintained at $37 \pm 0.5{ }^{\circ} \mathrm{C}$ by a feedback-controlled heating blanket. The head was fixed to a custom-built head plate with cyanoacrylic glue and stabilized with dental cement. Ophthalmic lubricant gel was applied to both eyes to prevent them from drying. After retracting the scalp, the area over the binocular region of the primary visual cortex was identified $(2.9-3.0 \mathrm{~mm}$ lateral from the midline and $0.5-0.7 \mathrm{~mm}$ rostral of lambda suture) and a small craniotomy ( 1-2 $\mathrm{mm}$ in diameter) performed. To allow for the insertion of recording electrodes a hole was made in the dura using a needle and fine forcipes and the exposed cortical surface was covered with a thin layer of $0.5-1 \%$ agarose. In vivo whole-cell recordings were obtained using the "blind" patch-clamp technique ${ }^{51}$. Electrodes were inserted into the brain at an oblique 
angle $\left(30-35^{\circ}\right)$ and lowered into the superficial layer of the cortex to stably record from layer $2 / 3$ neurons in the binocular region. Subpial depths ranged from 80 to $230 \mu \mathrm{m}$ as estimated

from the distance the micro-manipulator had advanced, taking into account the angle of insertion (average subpial depth $160.1 \pm 5.5 \mu \mathrm{m}, \mathrm{n}=81$ cells). Whole-cell current-clamp recordings were made with a current-clamp amplifier (BVC-700, Dagan Corp.) using glass pipettes (4-6 M $\Omega$ ) filled with a solution containing (in $\mathrm{mM}$ ): $130 \mathrm{~K}$-gluconate, $10 \mathrm{KCl}, 10$ HEPES, 4 MgATP, $0.3 \mathrm{Na}_{2} \mathrm{GTP}, 15 \mathrm{Na}_{2}$ Phosphocreatine (7.25-7.30 pH; 290-300 mOsm). Biocytin (0.2-0.5\%) was added to the solution for visualization and histological identification of recorded neurons. Whole-cell voltage-clamp recordings were made with a patch-clamp amplifier (Axopatch 200B) using pipettes (3-5 M 2 ) filled with the following solution (in $\mathrm{mM}$ ): 130 Cs-methansulfonate, $3 \mathrm{CsCl}, 10$ HEPES, 2 MgATP, $0.3 \mathrm{Na}_{2} \mathrm{GTP}, 10 \mathrm{Na}_{2}$ Phosphocreatine, 0.3 EGTA, 1 QX-314, (pH 7.25-7.30 with CsOH; 290-300 mOsm) and biocytin (0.2-0.5\%). Current and voltage signals were acquired at $50 \mathrm{kHz}$ by a Macintosh computer running Axograph acquisition software (Molecular Devices) using an ITC-18 interface (Instrutech). At the end of electrophysiology recording mice were perfused transcardially with $0.1 \mathrm{M}$ PBS followed by a solution of $4 \%$ paraformaldehyde (PFA). The brain was removed from the skull and kept in PFA overnight. Coronal slices (100 $\mu \mathrm{m}$ thick) of the visual cortex were prepared and processed with the avidin-biotin peroxidase reaction (Vectastain ABC kit, Vector laboratories). Slices were mounted in Moviol and cells reconstructed with the aid of a threedimensional computerized system (Neurolucida, Microbrightfield Inc., see ${ }^{52}$ ).

\section{Visual Stimulation}

Visual stimuli were generated in Matlab using the Psychophysics Toolbox extension and consisted of sinusoidal gratings. Gratings were presented at a temporal frequency of $2 \mathrm{~Hz}$, whereas the spatial frequency was optimized for each neuron within the range of 0.01-0.16 
cycles/degree. Each stimulus was presented for $1.5 \mathrm{~s}$ and preceded by a $2.5 \mathrm{~s}$ period of a blank screen with the same mean luminance as the stimulus. To study the dependence of binocular integration on stimulus orientation, direction and contrast, sinusoidal gratings drifting at 12 equally spaced directions $\left(0^{\circ}-330^{\circ}\right)$ were presented in a semi-random manner to a circular patch of diameter $60^{\circ}$ displayed on a CRT monitor with gamma correction $(60 \mathrm{~Hz}$ refresh rate, $30 \mathrm{~cd} / \mathrm{m} 2$ mean luminance) placed $25 \mathrm{~cm}$ in front of the mouse subtending the binocular visual space. The same stimulus was presented to each eye separately or together using computer-controlled motorized eye shutters. In experiments testing the dependence of binocular integration on inter-ocular phase disparity, mirrors were placed in front of each eye at 45 degrees and independently controlled visual stimuli presented to separate LCD screens located on either side of the animal at a distance of $25 \mathrm{~cm}$. Stimulus inter-ocular phase disparity was varied by keeping the initial phase of the grating presented to one eye fix and by changing the initial phase of the grating presented to the other eye in $60^{\circ}$ steps over the full range of $360^{\circ}$. Binocular phase disparity and stimulation condition (stimulation of the contralateral eye, ipsilateral eye or both eyes) were alternated semi-randomly. Visual stimulation were repeated 3 to 8 times for each neuron and averaged. Gratings were displayed at $100 \%$ contrast except in experiments on the contrast-dependence of binocular integration in which stimuli were presented at 3 different contrasts (30, 50 and 100\%).

\section{Data Analysis}

Cell classification. Recorded cells were classified as pyramidal neurons based on morphological and/or electrophysiological properties. Current-clamp experiments were performed on 65 cells. For each cell we quantified the evoked spike rate (R) in response to each grating direction $(\theta)$ as the average firing rate during the $1.5 \mathrm{~s}$ period of stimulus presentation minus the average spontaneous firing rate measured during the $1.5 \mathrm{~s}$ period 
before the stimulus. For each stimulation condition, the preferred direction was defined as the direction that evoked the highest spike rate ( $\left.\mathrm{R}_{\text {pref }}\right)$. In 34 cells we observed an increase in firing during presentation of the optimal stimulus to the contralateral and to the ipsilateral eye alone (Supplementary Fig. 2g). These cells were classified as binocular and were included in the analysis. The remaining cells either did not show visually-evoked suprathreshold responses or responded exclusively to either contralateral or ipsilataral eye stimulation. We classified binocular neurons as simple or complex cells based on their firing in response to drifting gratings at the preferred orientation presented to the contralateral eye as reported previously19,53 (Supplementary Fig 2a,b).

Voltage-clamp experiments were performed on 16 cells. The neuron's preferred direction was estimated as the one that evoked the largest excitatory postsynaptic current (EPSC) when holding the cell at $-80 \mathrm{mV}^{30,31}$. In 12 cells we measured a visually-evoke EPSC during presentation of the optimal stimulus to the contralateral (average EPSC peak amplitude=$159.2 \pm 35.3 \mathrm{pA}$ ) as well as the ipsilateral eye (average EPSC peak amplitude $=-109.8 \pm 24.9$ pA). These cells were classified as binocular and were included in the analysis.

Tuning properties. Evoked spike rates across all stimulus directions were used to determine the orientation tuning properties of the neuron during stimulation of the contralateral or ipsilateral eye alone (Supplementary Fig. 2) and for the simultaneous stimulation of both eyes together (Fig. 8). The neuron's preferred orientation, direction selectivity index (DSI), orientation tuning width (half-width at half height, HWHH) and ocular dominance index (ODI) were determined as reported previously 27,28 . The orientation selectivity index (OSI) was computed as 1 minus the circular variance ${ }^{30,54}$. A neuron that responds exclusively to a single orientation will have an OSI $=1$, whereas neurons that respond equally to all orientations will 
have OSI $=0$. Calculation of the OSI based on the spike rate at the preferred and orthogonal $\left(R_{\text {orth }}\right)$ directions using $\left(R_{\text {pref }}-R_{o r t h}\right) /\left(R_{p r e f}+R_{o r t h}\right)^{28}$ gave similar results. Matching of the preferred orientation across stimulation conditions was computed as 1 - the circular variance with the circular variance being calculated on the preferred orientation determined separately during stimulation of each eye alone and for stimulation of both eyes together. Values close to 1 indicate perfect matching.

Synaptic responses. To study how binocular neurons integrate synaptic inputs from the contralateral and ipsilateral eye, voltage traces recorded in current-clamp were processed to obtain an estimate of the overall synaptic response that underlies action potential generation. This was achieved by applying a sliding median filter of $10 \mathrm{~ms}$ width to all raw traces in order to selectively remove action potentials while preserving the overall sub-threshold membrane potential dynamics (Supplementary Fig. 1A-C). Median-filtered traces were then averaged over a single stimulus cycle ( 1 cycle has $0.5 \mathrm{~s}$ duration at a temporal frequency of $2 \mathrm{~Hz}$ ) and across stimulus repetitions ${ }^{29}$. We then fitted the average single cycle response with a $2 \mathrm{~Hz}$ sinusoidal function from which we measured the following parameters: the mean component $\left(\mathrm{V}_{\text {mean }}\right)$ quantified as the average potential over the stimulus presentation period relative to a $1.5 \mathrm{~s}$ baseline period before the stimulus, the modulation component $\left(\mathrm{V}_{\text {modulation }}\right)$ defined as the amplitude of the fitted sinusoid and the peak response $\left(\mathrm{V}_{\text {peak }}\right)$ defined as the sum of the mean and modulation components. Analysis performed on supra- and sub-threshold responses to visual stimulation revealed a negligible impact of median filtering on the quantification of $V_{\text {mean }}, V_{\text {modulation }}$ and $V_{\text {peak }}$ (Supplementary Fig. 1D-H).

Binocular integration. To investigate how the different components of synaptic responses summate during binocular stimulation we compared the observed response when the two 
eyes are stimulated together to the linear sum of the responses to stimulation of each eye separately. Residuals from linearity are calculated as the difference between the observed response during binocular stimulation and the expected linear sum of responses to stimulation of each eye alone. To measure the linearity and residuals from linearity as a continuous function over the period of stimulation traces were binned in $10 \mathrm{~ms}$ epochs and a point-by-point analysis performed for each time bin.

Synaptic current and conductance estimates. Currents evoked by the presentation of sinusoidal gratings were recorded during somatic whole-cell voltage-clamp without compensation for series resistance $\left(R_{s}\right)$ at two holding potentials $\left(V_{h}\right)$. Neurons were voltage-clamped at the predicted reversal potential for inhibition $(-80 \mathrm{mV}, \mathrm{n}=12$ cells $)$ to record EPSCs, and close to the reversal potential for excitation (average: $25.5 \pm 1.6 \mathrm{mV}, \mathrm{n}=10$ cells) to record inhibitory postsynaptic currents (IPSCs). Series resistance, assessed from the amplitude of the capacitance transient at the onset of a voltage step, was on average $35.1 \pm 2.8 \mathrm{M} \Omega(\mathrm{n}=12$ cells). Excitatory and inhibitory conductances were estimated as previously described ${ }^{32,33}$, after correction for the junction potential $(\sim 10 \mathrm{mV})$ and series resistance and assuming a reversal potential for excitation and inhibition of $0 \mathrm{mV}$ and $-80 \mathrm{mV}$, respectively.

Non-linear threshold model. The relationship between the membrane potential and spike rate (R) was described with the following 2-parameter model (Priebe 2004):

$$
\mathrm{R}\left(\mathrm{V}_{\mathrm{m}}\right)=\mathrm{k}^{*}\left[\mathrm{~V}_{\mathrm{m}}-\mathrm{V}_{\text {rest }}\right]^{\mathrm{p}_{+}}
$$

where $V_{m}$ is the membrane potential during the stimulus presentation and $V_{\text {rest }}$ is the resting membrane potential during baseline conditions. $\mathrm{k}$ and $\mathrm{p}$ are free parameters, corresponding to a gain factor and a power-law exponent, respectively, and were determined based on leastsquare fitting. The subscript “+” indicates rectification (membrane potential values below 
zero were set to zero). The fitted parameters were used to predict the firing rate associated with linear integration of binocular synaptic inputs.

\section{Neuron Model}

Computer simulations were performed using the NEURON simulation environment on a Linux desktop computer running Ubuntu 12.04 LTS. A multi-compartment model was obtained by reconstructing a biocytin-filled layer $2 / 3$ pyramidal neuron. The reconstructed neuronal model consisted of 136 compartments, subdivided into a total of 1021 segments. All dendritic branch diameters were scaled by a factor of 1.3 to account for cell shrinkage. Specific membrane resistance $\left(R_{m}\right)$, capacitance $\left(C_{m} ; 1 \mu F / \mathrm{cm}^{2}\right)$ and internal resistance $\left(R_{i} ; 100 \Omega / c m\right)$ were uniformly distributed throughout the model. In current clamp simulations the resting membrane potential was set to $-70 \mathrm{mV}$ and $\mathrm{R}_{\mathrm{m}}$ to $8000 \Omega / \mathrm{cm}^{2}$, giving an apparent membrane time constant ( $8 \mathrm{~ms}$ ) and input resistance ( $80 \mathrm{M} \Omega$ ), similar to that observed for this cell at the resting membrane potential in vivo. In voltage-clamp simulations the resting membrane potential was set to $-52 \mathrm{mV}$ to match holding currents recorded at hyperpolarized and depolarized potentials in these experiments. Spines were incorporated into the model by decreasing $R_{m}$ and increasing $C_{m}$ by a factor of 2 in distal dendritic compartments $(>40 \mu \mathrm{m}$ from the soma). No voltage-gated ion channels were included in the model. The series resistance of the somatic voltage-clamp "electrode" in voltage-clamp simulations was set to 35 $\mathrm{M} \Omega$ to match the average series resistance in experimental voltage-clamp recordings.

In models with dendritic inhibition 1000 excitatory and 300 inhibitory synapses were distributed randomly throughout the basal dendrites, with the density of inputs on a selected segment proportional to its surface area. Models with somatic inhibition contained 800 excitatory synapses distributed throughout the basal dendrites plus 180 inhibitory synapses 
at the soma. Excitatory inputs representing contralateral and ipsilateral inputs were sampled from the same pool (common pool model) or segregated into two pools with $60 \%$ classified as contralateral and 40\% classified as ipsilateral (segregated model). As seen in the Results there was essentially no difference between common pool and segregated models. The conductance change at excitatory synapses had an exponential rise and decay of 0.2 and 2 ms, respectively, a peak of $150 \mathrm{pS}$ and a reversal potential of $0 \mathrm{mV}$. Using these parameters the average unitary EPSP amplitude at the soma during activation of inputs randomly distributed throughout basal dendrites was $0.164 \pm 0.018 \mathrm{mV}$, consistent with previous experimental findings ${ }^{55}$. The conductance change at inhibitory synapses had an exponential rise and decay of 0.2 and $10 \mathrm{~ms}$, respectively, a peak of $150 \mathrm{pS}$ and a reversal potential of $-80 \mathrm{mV}$. In simulations with current-based synapses "excitatory" inputs had an amplitude of -2.8 pA, whereas "inhibitory" inputs had an amplitude of $+1.1 \mathrm{pA}$ in the models with dendritic inhibition and +0.7 pA in models with somatic inhibition, with only excitatory inputs activated at hyperpolarized potentials and only inhibitory inputs activated at depolarized potentials. To simulate the response to drifting gratings, synapses were activated in a sinusoidal manner ( 2 $\mathrm{Hz}$ modulation) by independent, non-homogeneous Poisson processes to match experimentally recorded currents and voltages during visual input. In the common pool model excitatory inputs were activated at rates between 0.54 (trough) and $27.54 \mathrm{~Hz}$ (peak) during contralateral input, between 0.36 and $18.36 \mathrm{~Hz}$ during ipsilateral input, and between 0.9 and $45.9 \mathrm{~Hz}$ during binocular input. In the segregated pool model the different sets of contralateral and ipsilateral synapses were activated at rates between 0.9 and $45.9 \mathrm{~Hz}$. The effective probability of release was 1.

In models with only excitatory input (Fig. 7) synapses were distributed on basal dendrites in different spatial configurations. In the dispersed configuration, contralateral and ipsilateral 
inputs were randomly distributed onto all dendritic branches. In the partitioned configuration, contralateral and ipsilateral inputs were distributed onto different dendritic branches such that no single branch contained both inputs. In the clustered configuration, all contralateral and ipsilateral inputs were positioned on the same, single dendritic branch. The number of synapses in the different models was 500 (dispersed), 550 (partitioned) and 700 (concentrated) and their activation was varied at rates between $1 \mathrm{~Hz}$ (trough) and $80 \mathrm{~Hz}$ (peak). Temporal correlations between synaptic inputs were introduced by using a common Poisson input train to drive sets of 50 synapses. This was the highest temporal correlation possible while still maintaining modulated responses similar to that seen experimentally. Common input trains were either restricted to within the contralateral and ipsilateral pools (monocular correlation) or shared between the pools (binocular correlation). In these simulations, the number and distribution of excitatory synapses was identical to that in the dispersed configuration.

\section{Statistics}

Statistical analysis used Prism 4.0 for Macintosh (GraphPad Softwares, Inc.). Average values are given as mean \pm s.e.m. Statistical comparison between two sets of matched data used a two-tailed paired t-test. When comparing three or more sets of data we used either a one-way or two-way ANOVA followed by a Bonferroni post-hoc multiple comparison test. A Chi squared test ( $\chi^{2}$ test) was used when comparing sets of data with categorical outcome as the variable and the Pearson's correlation coefficient was used when testing for significant correlations between two variables. All data sets were tested for normality. Significance was set at $p<0.05$. 


\section{References}

1. Koch, C., Poggio, T. \& Torre, V. Nonlinear interactions in a dendritic tree: localization, timing, and role in information processing. Proceedings of the National Academy of Sciences of the United States of America 80, 2799-2802 (1983).

2. Larkum, M.E., Zhu, J.J. \& Sakmann, B. A new cellular mechanism for coupling inputs arriving at different cortical layers. Nature 398, 338-341 (1999).

3. Losonczy, A. \& Magee, J.C. Integrative properties of radial oblique dendrites in hippocampal CA1 pyramidal neurons. Neuron 50, 291-307 (2006).

4. Schiller, J., Major, G., Koester, H.J. \& Schiller, Y. NMDA spikes in basal dendrites of cortical pyramidal neurons. Nature 404, 285-289 (2000).

5. Schiller, J., Schiller, Y., Stuart, G. \& Sakmann, B. Calcium action potentials restricted to distal apical dendrites of rat neocortical pyramidal neurons. The Journal of physiology 505 ( Pt 3), 605-616 (1997).

6. Stuart, G., Schiller, J. \& Sakmann, B. Action potential initiation and propagation in rat neocortical pyramidal neurons. The Journal of physiology 505 ( Pt 3), 617-632 (1997).

7. Williams, S.R. \& Stuart, G.J. Dependence of EPSP efficacy on synapse location in neocortical pyramidal neurons. Science 295, 1907-1910 (2002).

8. Polsky, A., Mel, B.W. \& Schiller, J. Computational subunits in thin dendrites of pyramidal cells. Nature neuroscience 7, 621-627 (2004).

9. Hoffman, D.A., Magee, J.C., Colbert, C.M. \& Johnston, D. K+ channel regulation of signal propagation in dendrites of hippocampal pyramidal neurons. Nature 387, 869-875 (1997).

10. Magee, J.C. \& Johnston, D. Synaptic activation of voltage-gated channels in the dendrites of hippocampal pyramidal neurons. Science 268, 301-304 (1995).

11. Stuart, G. \& Sakmann, B. Amplification of EPSPs by axosomatic sodium channels in neocortical pyramidal neurons. Neuron 15, 1065-1076 (1995).

12. Magee, J.C. Dendritic lh normalizes temporal summation in hippocampal CA1 neurons. Nat. Neurosci. 2, 508-514 (1999).

13. Murayama, M., et al. Dendritic encoding of sensory stimuli controlled by deep cortical interneurons. Nature 457, 1137-1141 (2009).

14. Palmer, L.M., et al. The Cellular Basis of GABAB-Mediated Interhemispheric Inhibition. Science 335, 989-993 (2012).

15. $\mathrm{Xu}, \mathrm{N} . L .$, et al. Nonlinear dendritic integration of sensory and motor input during an active sensing task. Nature 492, 247-251 (2012).

16. Lavzin, M., Rapoport, S., Polsky, A., Garion, L. \& Schiller, J. Nonlinear dendritic processing determines angular tuning of barrel cortex neurons in vivo. Nature 490, 397-401 (2012).

17. Cash, S. \& Yuste, R. Input summation by cultured pyramidal neurons is linear and position-independent. The Journal of neuroscience : the official journal of the Society for Neuroscience 18, 10-15 (1998).

18. Cash, S. \& Yuste, R. Linear summation of excitatory inputs by CA1 pyramidal neurons. Neuron 22, 383-394 (1999).

19. Priebe, N.J., Mechler, F., Carandini, M. \& Ferster, D. The contribution of spike threshold to the dichotomy of cortical simple and complex cells. Nat Neurosci 7, 1113-1122 (2004).

20. Chen, X., Leischner, U., Rochefort, N.L., Nelken, I. \& Konnerth, A. Functional mapping of single spines in cortical neurons in vivo. Nature 475, 501-505 (2011). 
21. Varga, Z., Jia, H., Sakmann, B. \& Konnerth, A. Dendritic coding of multiple sensory inputs in single cortical neurons in vivo. Proceedings of the National Academy of Sciences 108, 15420-15425 (2011).

22. Kleindienst, T., Winnubst, J., Roth-Alpermann, C., Bonhoeffer, T. \& Lohmann, C. Activitydependent clustering of functional synaptic inputs on developing hippocampal dendrites. Neuron 72, 1012-1024 (2011).

23. Takahashi, N., et al. Locally Synchronized Synaptic Inputs. Science 335, 353-356 (2012).

24. Ohzawa, I. \& Freeman, R.D. The binocular organization of complex cells in the cat's visual cortex. J Neurophysiol 56, 243-259 (1986).

25. Ohzawa, I. \& Freeman, R.D. The binocular organization of simple cells in the cat's visual cortex. J Neurophysiol 56, 221-242 (1986).

26. Jagadeesh, B., Wheat, H.S., Kontsevich, L.L., Tyler, C.W. \& Ferster, D. Direction selectivity of synaptic potentials in simple cells of the cat visual cortex. J Neurophysiol 78, 2772-2789 (1997).

27. Wang, B.S., Sarnaik, R. \& Cang, J. Critical period plasticity matches binocular orientation preference in the visual cortex. Neuron 65, 246-256 (2010).

28. Niell, C.M. \& Stryker, M.P. Highly selective receptive fields in mouse visual cortex. $J$ Neurosci 28, 7520-7536 (2008).

29. Carandini, M. \& Ferster, D. Membrane potential and firing rate in cat primary visual cortex. J Neurosci 20, 470-484 (2000).

30. Tan, A.Y., Brown, B.D., Scholl, B., Mohanty, D. \& Priebe, N.J. Orientation selectivity of synaptic input to neurons in mouse and cat primary visual cortex. J Neurosci 31, 12339-12350 (2011).

31. Atallah, B.V., Bruns, W., Carandini, M. \& Scanziani, M. Parvalbumin-expressing interneurons linearly transform cortical responses to visual stimuli. Neuron $\mathbf{7 3}, \mathbf{1 5 9}$ 170 (2012).

32. Borg-Graham, L.J., Monier, C. \& Fregnac, Y. Visual input evokes transient and strong shunting inhibition in visual cortical neurons. Nature 393, 369-373 (1998).

33. Cruikshank, S.J., Lewis, T.J. \& Connors, B.W. Synaptic basis for intense thalamocortical activation of feedforward inhibitory cells in neocortex. Nat Neurosci 10, 462-468 (2007).

34. Medini, P. Layer- and cell-type-specific subthreshold and suprathreshold effects of long-term monocular deprivation in rat visual cortex. The Journal of neuroscience : the official journal of the Society for Neuroscience 31, 17134-17148 (2011).

35. Restani, L., et al. Functional masking of deprived eye responses by callosal input during ocular dominance plasticity. Neuron 64, 707-718 (2009).

36. Williams, S.R. \& Mitchell, S.J. Direct measurement of somatic voltage clamp errors in central neurons. Nature neuroscience 11, 790-798 (2008).

37. Branco, T. \& Häusser, M. Synaptic integration gradients in single cortical pyramidal cell dendrites. Neuron 69, 885-892 (2011).

38. Krueppel, R., Remy, S. \& Beck, H. Dendritic integration in hippocampal dentate granule cells. Neuron 71, 512-528 (2011).

39. Enoki, R., Namiki, M., Kudo, Y. \& Miyakawa, H. Optical monitoring of synaptic summation along the dendrites of CA1 pyramidal neurons. Neuroscience 113, 10031014 (2002).

40. Jaubert-Miazza, L., et al. Structural and functional composition of the developing retinogeniculate pathway in the mouse. Vis Neurosci 22, 661-676 (2005). 
41. Muir-Robinson, G., Hwang, B.J. \& Feller, M.B. Retinogeniculate axons undergo eyespecific segregation in the absence of eye-specific layers. J Neurosci 22, 5259-5264 (2002).

42. Ziburkus, J. \& Guido, W. Loss of binocular responses and reduced retinal convergence during the period of retinogeniculate axon segregation. J Neurophysiol 96, 2775-2784 (2006).

43. Jia, H., Rochefort, N.L., Chen, X. \& Konnerth, A. Dendritic organization of sensory input to cortical neurons in vivo. Nature 464, 1307-1312 (2010).

44. Yizhar, 0., et al. Neocortical excitation/inhibition balance in information processing and social dysfunction. Nature 477, 171-178 (2011).

45. Haider, B. \& McCormick, D.A. Rapid neocortical dynamics: cellular and network mechanisms. Neuron 62, 171-189 (2009).

46. DeAngelis, G.C., Ohzawa, I. \& Freeman, R.D. Depth is encoded in the visual cortex by a specialized receptive field structure. Nature 352, 156-159 (1991).

47. Anzai, A., Ohzawa, I. \& Freeman, R.D. Neural mechanisms for encoding binocular disparity: receptive field position versus phase. J Neurophysiol 82, 874-890 (1999).

48. Ohzawa, I., DeAngelis, G.C. \& Freeman, R.D. Stereoscopic depth discrimination in the visual cortex: neurons ideally suited as disparity detectors. Science 249, 1037-1041 (1990).

49. Wilson, N.R., Runyan, C.A., Wang, F.L. \& Sur, M. Division and subtraction by distinct cortical inhibitory networks in vivo. Nature 488, 343-348 (2012).

50. Lee, S.H., et al. Activation of specific interneurons improves V1 feature selectivity and visual perception. Nature 488, 379-383 (2012).

51. Margrie, T.W., Brecht, M. \& Sakmann, B. In vivo, low-resistance, whole-cell recordings from neurons in the anaesthetized and awake mammalian brain. Pflugers Archiv: European journal of physiology 444, 491-498 (2002).

52. Kole, M.H., Brauer, A.U. \& Stuart, G.J. Inherited cortical HCN1 channel loss amplifies dendritic calcium electrogenesis and burst firing in a rat absence epilepsy model. The Journal of physiology 578, 507-525 (2007).

53. Skottun, B.C., et al. Classifying simple and complex cells on the basis of response modulation. Vision research 31, 1079-1086 (1991).

54. Ringach, D.L., Shapley, R.M. \& Hawken, M.J. Orientation selectivity in macaque V1: diversity and laminar dependence. The Journal of neuroscience : the official journal of the Society for Neuroscience 22, 5639-5651 (2002).

55. Silver, R.A., Lubke, J., Sakmann, B. \& Feldmeyer, D. High-probability uniquantal transmission at excitatory synapses in barrel cortex. Science 302, 1981-1984 (2003). 


\section{Acknowledgments}

We are grateful to Dr. Samuel Solomon for discussions. We would also like to thank Tobias Bock for help with Matlab programming. This work was supported by the Swiss National Science Foundation, the Australian National Health and Medical Research Council and the John Curtin School of Medical Research.

\section{Author Contributions}

GJS and FL conceived the project. FL conducted the experiments and performed all analysis.

KI contributed to early experiments. MST performed all simulations. All authors discussed the data and contributed to writing the manuscript.

\section{Competing Financial Interests}

The authors declare no competing financial interests. 


\section{Figure Legends}

Figure 1. Summation of binocular inputs at the preferred orientation. (a) Top: Schematic of in vivo whole-cell recording in mouse visual cortex during presentation of the same visual stimulus selectively to the ipsilateral (left) or contralateral eye (middle) and to both eyes together (right) using computer-controlled eye shutters. Bottom: Three overlaid trials of membrane potential during baseline (grey bar, top) and in response to drifting gratings at the preferred orientation (dashed bars, top) during stimulation of the ipsilateral (left) or contralateral eye (middle) and both eyes together (right) together with the corresponding color-coded average response after median filtering. The dashed horizontal grey line indicates the average membrane potential during baseline. (b) Single-cycle average of a median-filtered synaptic potential response (light green) fitted with a sinusoidal function (black). The peak $\left(\mathrm{V}_{\text {peak }}\right)$, mean $\left(\mathrm{V}_{\text {mean }}\right)$ and modulation component $\left(\mathrm{V}_{\text {modulation }}\right)$ of the response are indicated. (c) Single-cycle synaptic response to stimulation of both eyes (green) superimposed with the linear sum of contralateral and ipsilateral responses (pink) for 3 simple cells with expected linear sums of different peak amplitude (left: < $15 \mathrm{mV}$; center: $\sim 15 \mathrm{mV}$; right: > $15 \mathrm{mV}$ ). Data

fitted with sinusoids shown in dark green and purple. (d) $V_{\text {peak }}$ of synaptic responses to stimulation of both eyes at the preferred orientation at either the preferred (blue) or nonpreferred direction (red) versus the corresponding expected linear sum (40 responses, n=20 cells). Dashed diagonal line indicates linear summation. (e) Same data as in $\mathbf{d}$ in $5 \mathrm{mV}$ bins for the expected linear sum and expressed as an average \pm s.e.m. ( $\mathrm{n}=20$ cells; ${ }^{*} p<0.05,{ }^{* *} p<0.01$, ${ }^{* * *} p<0.001$, two-way ANOVA, Bonferroni post-test). (f) Plot of the residuals from linearity (difference between observed $V_{\text {peak }}$ and expected $V_{\text {peak }}$ ) versus the expected linear sum. Only the residuals for large expected linear sums ( $V_{\text {peak }}>15 \mathrm{mV} ; 18$ responses, $n=10$ simple cells) are shown. Data were fitted with a linear regression (black line). $r$ and $p$ values indicate the Pearson's correlation coefficient and the significance of correlation, respectively. 


\section{Figure 2. Dependence of binocular integration on stimulus orientation and response}

phase. (a) Single-cycle responses during stimulation of both eyes at 6 different orientations (light green) together with the corresponding expected linear sum of contralateral and ipsilateral responses (pink). The preferred orientation is indicated as $0^{\circ}$. Sinusoidal fits are shown in dark green and purple. (b) Average $V_{\text {peak }}( \pm$ s.e.m.) of responses to stimulation of both eyes (green symbols) together with the expected linear sum (purple symbols) plotted as a function of stimulus orientation (40 responses for each stimulus orientation, $\mathrm{n}=20$ cells; ${ }^{*} p<$ 0.05, two-way ANOVA, Bonferroni post-test). Data fitted with a Gaussian function. (c) Average $V_{\text {peak }}( \pm$ s.e.m.) of responses to stimulation of both eyes color coded for different stimulus orientations and plotted versus the corresponding expected linear sum in $5 \mathrm{mV}$ bins. Dashed diagonal line indicates linear summation. (d) Average residuals from linearity ( \pm s.e.m.) for the different stimulus orientations (same color code as in c). Responses were sorted based on the amplitude of the expected linear sum (left: $V_{\text {peak }}$ linear sum $<15 \mathrm{mV}$; right: $\mathrm{V}_{\text {peak }}$ linear sum $>15 \mathrm{mV}$ ). The number of responses in each group is indicated in brackets $\left(^{*} p<0.01\right.$; ns: not significant, two-way ANOVA, Bonferroni post-test). (e) Top: Single-cycle responses to stimulation of the contralateral (blue) or ipsilateral eye (red) in different cells showing different degrees of phase difference. Bottom: Single-cycle responses to stimulation of both eyes (green) together with the expected linear sum (pink) for the responses showed in the top. Data fitted with sinusoids. (f) Average $V_{\text {peak }}( \pm$ s.e.m.) of responses to stimulation of both eyes (green symbols) together with the expected linear sum (pink symbols) plotted as a function of the phase difference between contralateral and ipsilateral responses. Response phase differences ranging from $0^{\circ}$ (in phase) to $180^{\circ}$ (out of phase) binned in $30^{\circ}$ bins. Data were fitted with a linear regression number of responses for each phase difference is indicated in brackets, $\mathrm{n}=20$ cells; ${ }^{*} p<0.05,{ }^{* *} p<0.01$, two-way ANOVA, Bonferroni post-test). (g) Average $V_{\text {peak }}( \pm$ s.e.m.) of responses to stimulation of both eyes color-coded for phase difference plotted versus the corresponding expected linear sum in $5 \mathrm{mV}$ bins. Dashed diagonal line indicates linear summation. (h) Average residuals from linearity ( \pm s.e.m.) for the different phase differences (same color code as in $\mathbf{g}$ ). Responses sorted based on the amplitude of the expected linear sum (left: $\mathrm{V}_{\text {peak }}$ linear sum $<15 \mathrm{mV}$; right: $\mathrm{V}_{\text {peak }}$ linear sum > $15 \mathrm{mV}$ ). The number of responses in each group is indicated in brackets $\left({ }^{*} p<0.05\right.$; ns: not significant, two-way ANOVA, Bonferroni post-test). 
Figure 3. Dependence of binocular integration on phase disparity. (a) Schematic of in vivo whole-cell recording in mouse visual cortex during presentation of different visual stimuli to each eye with a haploscope. (b) Top: Example of monocular stimulation with drifting gratings at the preferred orientation in which the initial phase of the stimulus to the ipsilateral eye is kept constant, whereas the initial phase of the stimulus to the contralateral eye is varied. Bottom: Single-cycle responses to stimulation of the ipsilateral (red) or contralateral (blue) eye showing different degrees of phase difference between monocular responses in a single cell. Data fitted with sinusoids. (c) Plot of the average relative phase \pm \pm s.e.m.) of the monocular synaptic response versus the initial phase of the variable stimulus ( $n=7$ contralateral eye; $n=4$ ipsilateral eye). In different cells, the monocular response to gratings with initial phase of $0^{\circ}$ has been assigned a relative phase of $0^{\circ}$. (d) Top: Binocular stimulation with stimuli showing different degrees of inter-ocular phase disparity. Bottom: Single-cycle responses during stimulation of both eyes at 6 different inter-ocular phase disparities (green) together with the corresponding expected linear sum of contralateral and ipsilateral responses (pink). The preferred phase disparity for this cell is $60^{\circ}$ (Same cell as in b). Sinusoidal fits are shown in dark green and purple. (e) Top: Plot of spike rate versus stimulus phase disparity for a representative simple cell (preferred phase disparity: $120^{\circ}$ ). Bottom: Plot of the phase difference between monocular synaptic responses versus the stimulus phase disparity (same cell as for the top). (f) Top: Summary plot of average spike rates ( \pm s.e.m.) normalized to the response at the preferred phase disparity (Pref.) versus the relative stimulus phase disparity. Bottom: Summary box plot of the average phase difference between the monocular synaptic responses versus the relative stimulus phase disparity $(\mathrm{n}=7$ cells; ${ }^{*} p<0.05,{ }^{* *} p<0.01$, one-way ANOVA, Bonferroni post-test). (g) Average $\mathrm{V}_{\text {peak }}( \pm$ s.e.m.) of responses to stimulation of both eyes (green symbols) and of the expected linear sum (pink symbols) plotted as a function of the relative stimulus phase disparity. Responses to $60^{\circ}$ and $300^{\circ}\left(-60^{\circ}\right)$ stimulus phase disparity or to $120^{\circ}$ and $240^{\circ}\left(-120^{\circ}\right)$ have been pooled. Data were fitted with a linear regression (11 cells; variable stimulus presented to either the contralateral $(\mathrm{n}=7)$ or ipsilateral eye $(\mathrm{n}=4) ;{ }^{*} p<0.05,{ }^{* *} p<0.01$, two-way ANOVA, Bonferroni post-test). 
Figure 4. Summation of different components of the synaptic response. (a) Single-cycle responses to drifting gratings presented to both eyes at the indicated direction (light green). The preferred direction is indicated as $0^{\circ}$. Data fitted with sinusoidal functions (dark green). Dashed horizontal lines indicate the $V_{\text {mean }}$ for each response. (b,c) Average amplitude of $\mathrm{V}_{\text {modulation }}$ (b) and $\mathrm{V}_{\text {mean }}$ (c) during stimulation of both eyes at 12 different directions (preferred direction: $0^{\circ} ; \mathrm{n}=20$ cells). (d) Orientation selectivity index of $\mathrm{V}_{\text {modulation }}$ and $\mathrm{V}_{\text {mean }}$. Bars represent mean and points show individual cells ( $\mathrm{n}=20$ cells; ${ }^{*} p<0.001$, paired $\mathrm{t}$-test). $(\mathbf{e}, \mathbf{f})$ Plots of $V_{\text {modulation }}(\mathbf{e})$ and $V_{\text {mean }}(\mathbf{f})$ during stimulation of both eyes plotted versus the corresponding expected linear sum (240 responses to 12 stimulus directions, $\mathrm{n}=20$ cells). Red symbols: $\mathrm{V}_{\text {peak }}$ linear sum < $15 \mathrm{mV}$ ( $\mathrm{n}=172$ responses); blue symbols: $\mathrm{V}_{\text {peak }}$ linear sum $>15$ $\mathrm{mV}$ ( $\mathrm{n}=68$ responses). Dashed diagonal line indicates linear summation. (g) Average residuals from linearity ( \pm s.e.m.) for $V_{\text {modulation }}$ and $V_{\text {mean }}$ for small (red bars) and large (blue bars) expected linear sums ( $\mathrm{n}=20$ cells; ${ }^{*} p<0.001$, two-way ANOVA, Bonferroni post-test). (h) Representative synaptic potentials (observed and expected liner sum) used for point-by-point analysis of sub-linear summation during a single response. (i) Plot of the residuals from linearity versus the expected linear sum for the response shown on the left after binning the synaptic potentials $\left(\mathrm{V}_{\mathrm{m}}\right)$ in $10 \mathrm{~ms}$ epochs. Data were fitted with a linear regression (black line). $r$ and $p$ values indicate the Pearson's correlation coefficient and the significance of correlation, respectively. Compare with Fig. 1f. (j) Pooled data during stimulation of both eyes together. $V_{\mathrm{m}}$ binned in $10 \mathrm{~ms}$ epochs. 12,000 time bins from 240 averaged responses to 12 stimulus directions in 20 simple cells. Small grey symbols indicate individual data points. Large open symbols indicate average \pm s.e.m after binning in $2.5 \mathrm{mV}$ increments. Dashed diagonal line indicates linear summation. 


\section{Figure 5. Recruitment and summation of excitation and inhibition during binocular}

integration. (a) EPSCs (green) recorded at $-80 \mathrm{mV}$ in response to stimulation of both eyes with drifting gratings at 6 different orientations. (b) Normalized EPSC amplitude \pm \pm s.e.m.; averaged over the duration of the stimulus) during stimulation of both eyes versus stimulus orientation (preferred orientation: $0^{\circ} ; \mathrm{n}=12$ cells). Data fit with a Gaussian. (c) Top: Singlecycle EPSC recorded at $-80 \mathrm{mV}$ in response to stimulation of both eyes at the preferred orientation (green) together with the linear sum of EPSCs evoked by stimulation of each eye alone (pink). Bottom: Plot of EPSC peak amplitude evoked by stimulation of both eyes with gratings at 4 different orientations (preferred, $+30^{\circ},+60^{\circ},+90^{\circ}$ ) versus the corresponding linear sum ( $n=44$ responses, $n=11$ cells). Dashed diagonal line indicates linear summation. (d) Top: Single-cycle IPSC recorded at $+20 \mathrm{mV}$ in response to stimulation of both eyes at the preferred orientation (green) together with the linear sum of IPSCs evoked by stimulation of each eye alone (pink). Same cell and stimulus as in c. Bottom: Plot of the IPSC peak amplitude evoked by stimulation of both eyes with gratings at 4 different orientations (preferred, $+30^{\circ}$, $\left.+60^{\circ},+90^{\circ}\right)$ versus the corresponding linear sum $(n=40$ responses, $n=10$ cells). Dashed diagonal line indicates linear summation. (e) Top: Single-cycle average of estimated excitatory ( $g_{e}$, blue) and inhibitory $\left(g_{i}\right.$, red) conductance changes in a single cell in response to gratings at the preferred orientation. Bottom: Summary plot of $g_{i}$ versus $g_{e}$ ( $n=9$ cells). Data from individual cells (represented by different colors) were binned at $0.5 \mathrm{nS}$ increments for $\mathrm{g}_{\mathrm{e}}$ and expressed as average \pm s.e.m. Colored lines represent linear fits to each data set. 


\section{Figure 6. Balanced recruitment of excitation and inhibition explains sub-linear}

integration of binocular synaptic inputs. (a) Models of binocular integration in layer $2 / 3$ (L2/3) pyramidal neurons in which contralateral (blue) and ipsilateral (red) inputs converge upstream from layer $2 / 3$, for example in layer 4 (L4, left) or arise via separate inputs, for example from layer 4 neurons with different ocular dominance (middle) or from different hemispheric pathways (right). Putative feed-forward interneurons (I) are shown in grey. (b) Top left: Location of excitatory synapses (green circles) on basal dendrites of a morphologically realistic model of a layer $2 / 3$ pyramidal neuron where contralateral and ipsilateral inputs activate the same set of synapses (Common-pool model). Top right: Dendritic location of excitatory synapses where contralateral (blue triangles) and ipsilateral (red circles) inputs activated different sets of synapses (Segregated model). Bottom left: Location of inhibitory synapses (orange circles) on basal dendrites in the model with dendritic inhibition. Bottom right: Location of inhibitory synapses (orange) in the model with somatic inhibition. (c) Top: Somatic voltage-clamp simulations of the single-cycle EPSC amplitude during activation of contralateral and ipsilateral inputs together compared to their linear sum using conductance-based synapses for the common-pool model with dendritic (light blue) or somatic (pink) inhibition, and for the segregated model with dendritic (dark blue) or somatic (orange) inhibition. Experimental data (green symbols, same as in Fig. 5c, bottom panel). Bottom: Somatic voltage-clamp simulations of the single-cycle IPSC amplitude during activation of contralateral and ipsilateral inputs together compared to their linear sum using conductance-based synapses. Same color code as for the top. Experimental data (green symbols, same as in Fig. 5d, bottom panel). (d) Same as (c) but using current-based synapses. (e) Current-clamp simulations of the single-cycle synaptic responses to activation of contralateral and ipsilateral inputs together (green) compared to their linear sum (pink) in the common-pool and segregated models with dendritic or somatic inhibition. (f) Plot of the synaptic response amplitude during activation of contralateral and ipsilateral inputs plotted versus the linear sum for all models (lines; same color code as in c). Experimental data (open symbols, same as in Fig. 4j). 


\section{Figure 7. Interactions between excitatory synapses alone cannot account for sub-linear}

integration of binocular inputs. (a) Dendritic location of contralateral (blue symbols) and ipsilateral (red symbols) excitatory synapses on the basal dendrites of a morphologically realistic model of a layer $2 / 3$ pyramidal neuron. Synapses were distributed in three different spatial configurations: dispersed (left), partitioned (middle) and concentrated (right). (b) Simulated single-cycle voltage responses recorded at the soma during activation of contralateral and ipsilateral inputs together (green) at different intensities to evoke responses of different amplitude for the dispersed (left), partitioned (middle) and concentrated (right) configurations. Corresponding expected linear sums (pink) are superimposed. (c) Plot of synaptic response amplitude during activation of contralateral and ipsilateral inputs together versus the corresponding expected linear sum for the dispersed (orange), partitioned (light blue) and concentrated (red) models together with experimental data (open symbols, from Fig. 4j). (d) Raster plots of uncorrelated (left) and correlated (right) input spike trains driving contralateral (blue) and ipsilateral (red) sets of synapses during binocular stimulation. Correlation was introduced by using common Poisson input trains to drive sets of 50 synapses. (e) Peristimulus time histograms (PSTHs) of the input spike trains shown in $\mathbf{d}$ averaged over six stimulation cycles for different time windows (2 ms, $5 \mathrm{~ms}, 10$ $\mathrm{ms}, 20 \mathrm{~ms}$ and $50 \mathrm{~ms}$ ) for uncorrelated (left, orange) and binocularly correlated (right, green) inputs. (f) Cross-correlogram (1 ms time bins) averaged across all pairs of input spike trains for the uncorrelated and binocularly correlated spike trains. (g) Plot of synaptic response amplitude during activation of contralateral and ipsilateral sets of inputs in the dispersed model versus the corresponding expected linear sums for the uncorrelated (orange), monocularly correlated (red) and binocularly correlated (green) models together with the experimental data (open symbols, from Fig. 4j). 
Figure 8. Impact of sub-linear binocular integration on action potential output. (a) Plot of the average spike count versus membrane potential (30 ms time bins) for one cell. Data were fitted to a threshold non-linearity model (black trace; $\mathrm{k}=1.16 \mathrm{E}-05, \mathrm{p}=4.46$; see $^{19}$ ). (b) Summary plot of average spike rates predicted using the threshold non-linearity model during stimulation of both eyes together versus experimentally observed spike rates (240 responses to 12 stimulus directions, $\mathrm{n}=20$ cells). Spike rate was calculated over the $1.5 \mathrm{~s}$ period of stimulus presentation. The dashed diagonal line indicates a perfect correlation between predicted and observed values. (c) PSTHs of the observed action potential output during stimulation of both eyes together at the preferred orientation (top left, $30 \mathrm{~ms}$ time bins) and corresponding synaptic response (bottom left), together with the predicted action potential output (top right, $30 \mathrm{~ms}$ time bins) for the same cell assuming linear sum of contralateral and ipsilateral synaptic responses (bottom right). (d) Pooled data on the observed spike rate during stimulation of both eyes together plotted versus the predicted spike rate assuming linear summation of synaptic responses to each eye alone (40 responses to preferred stimuli, $n=20$ cells). (e) Summary of observed (green symbols) and predicted (pink symbols) spike rates as a function of the expected linear sum of $\mathrm{V}_{\text {peak }}$ (in $5 \mathrm{mV}$ bins). Data fitted with the same function as used in a to describe the relationship between membrane potential and spike output. (f) Summary of observed (green symbols) and predicted (pink symbols) spike rates as a function of the linear sum of the spike rates evoked by stimulation of each eye separately. Data fitted with a linear function (slope $=1.02 \pm 0.06$ for observed spike rates, dark green line; slope $=2.12 \pm 0.24$ for predicted spike rates, purple line; $\mathrm{p}<0.0001$ ). Dashed diagonal line indicates linear summation of spike rate. (g) Average tuning curves based on the observed (green symbols) and predicted (pink symbols) spike rates during presentation of gratings at different orientations to both eyes. Data fitted with a Gaussian function. (h,i,j) Plots of the direction selectivity index (DSI, h), tuning width (HMHH, i) and orientation selectivity index (OSI, j) observed during stimulation of both eyes (green) together with that predicted assuming linear summation of ipsilateral and contralateral inputs (pink). Bars represent mean, and points show individual cells ( $\mathrm{n}=20$ simple cells; ${ }^{*} p<$ 0.05 , paired t-test). 
a

Ipsilateral eye
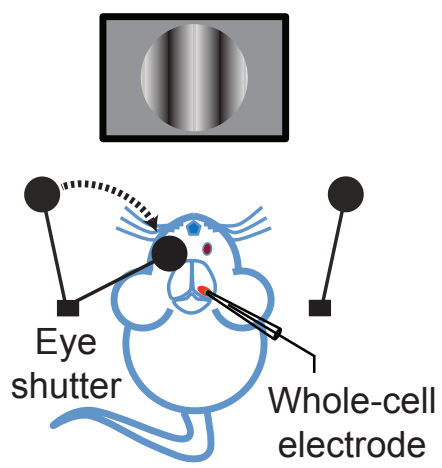

Baseline Stimulus

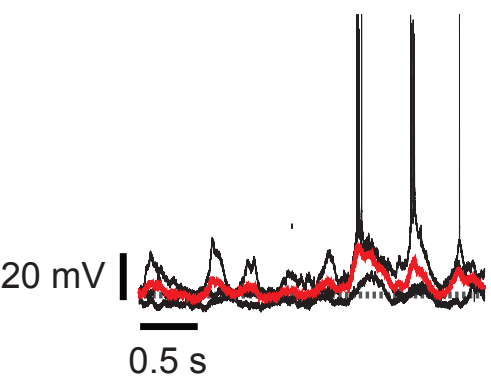

b

Synaptic response single-cycle average

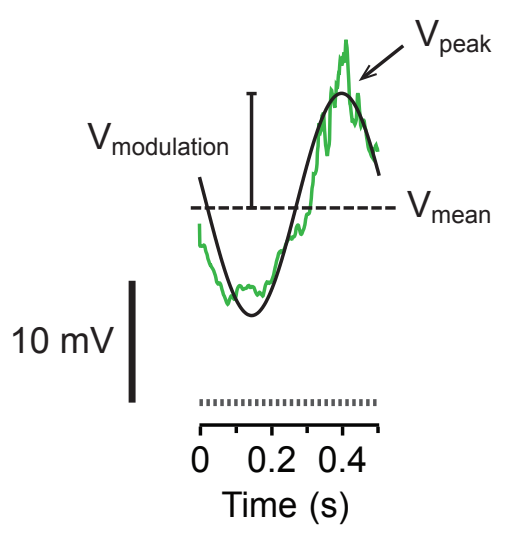

d

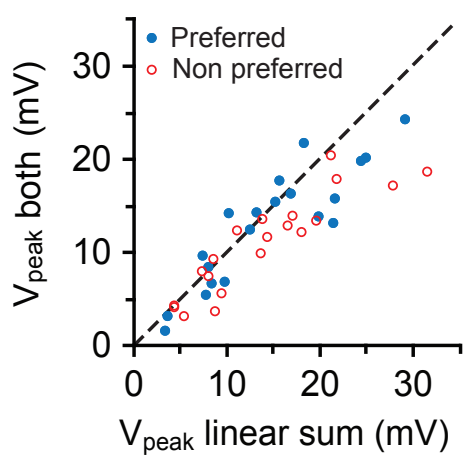

Contralateral eye
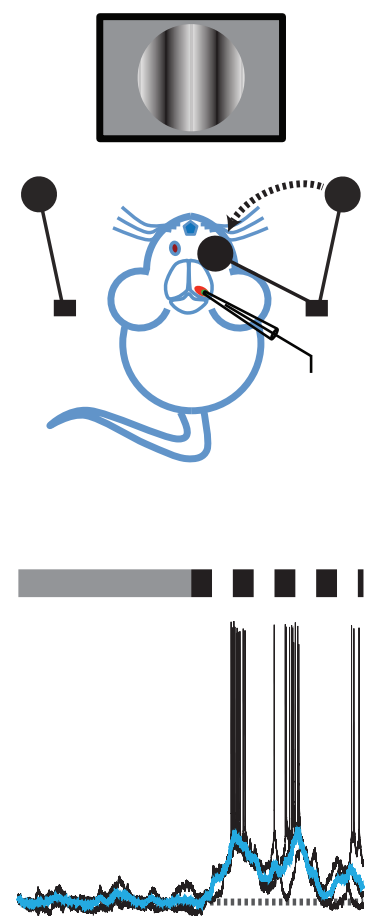

C

Cell 1

Cell 2

Cell 3

Linear sum $<15 \mathrm{mV}$ Linear sum $\sim 15 \mathrm{mV} \quad$ Linear sum $>15 \mathrm{mV}$
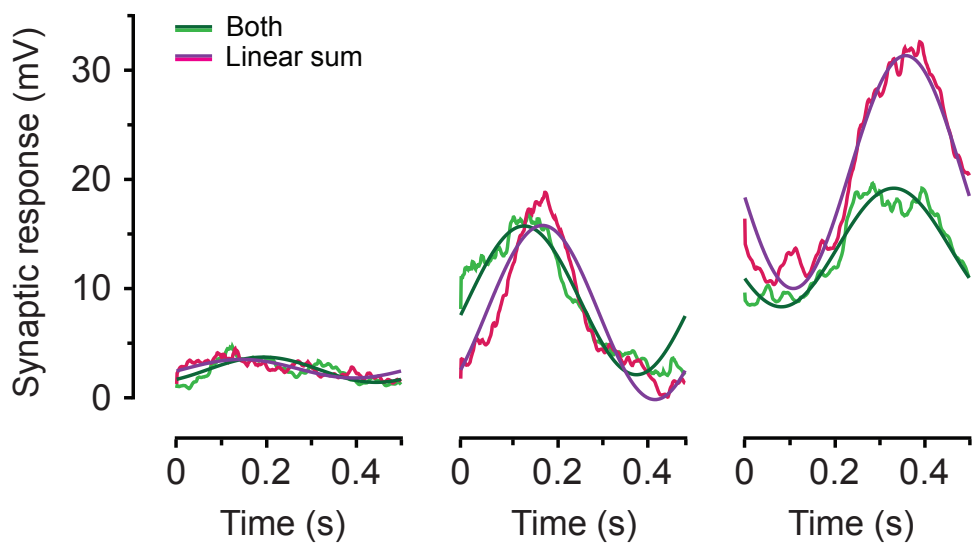

e

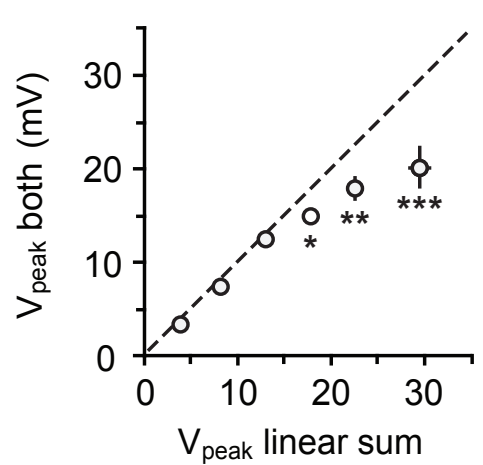


a

Normalized orientation
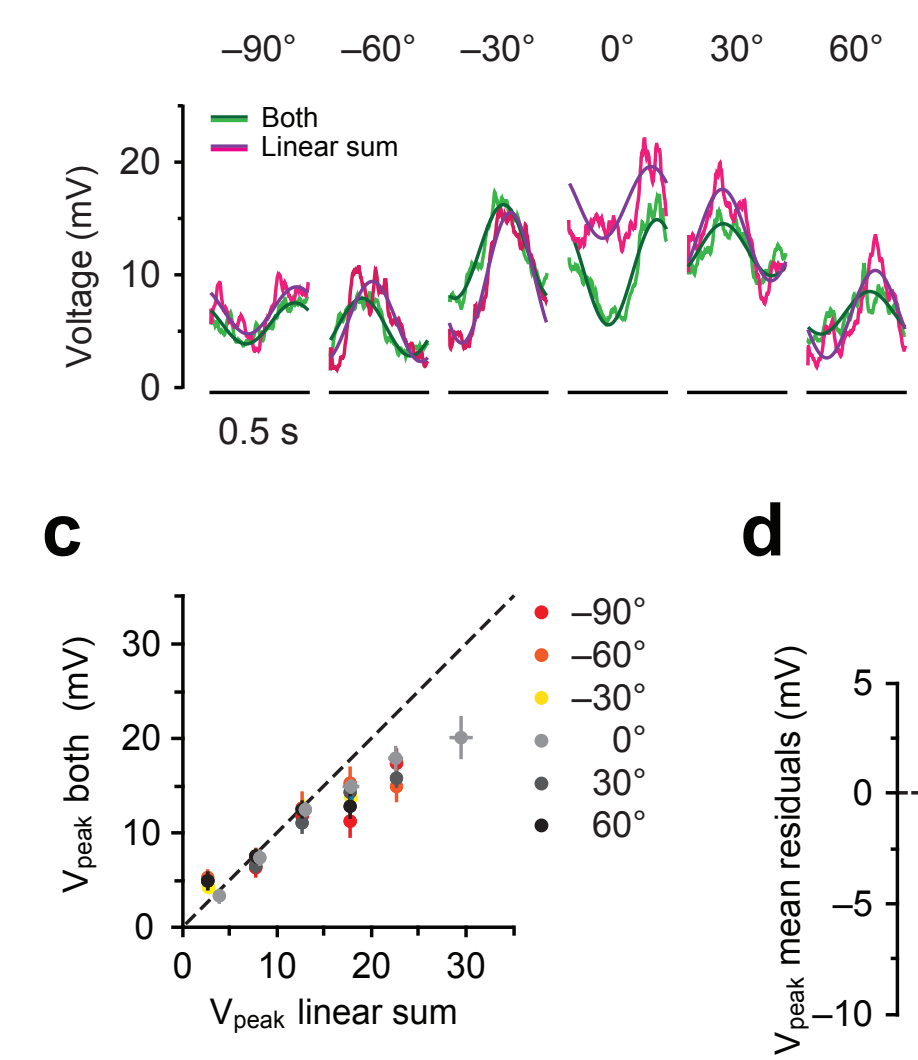

d
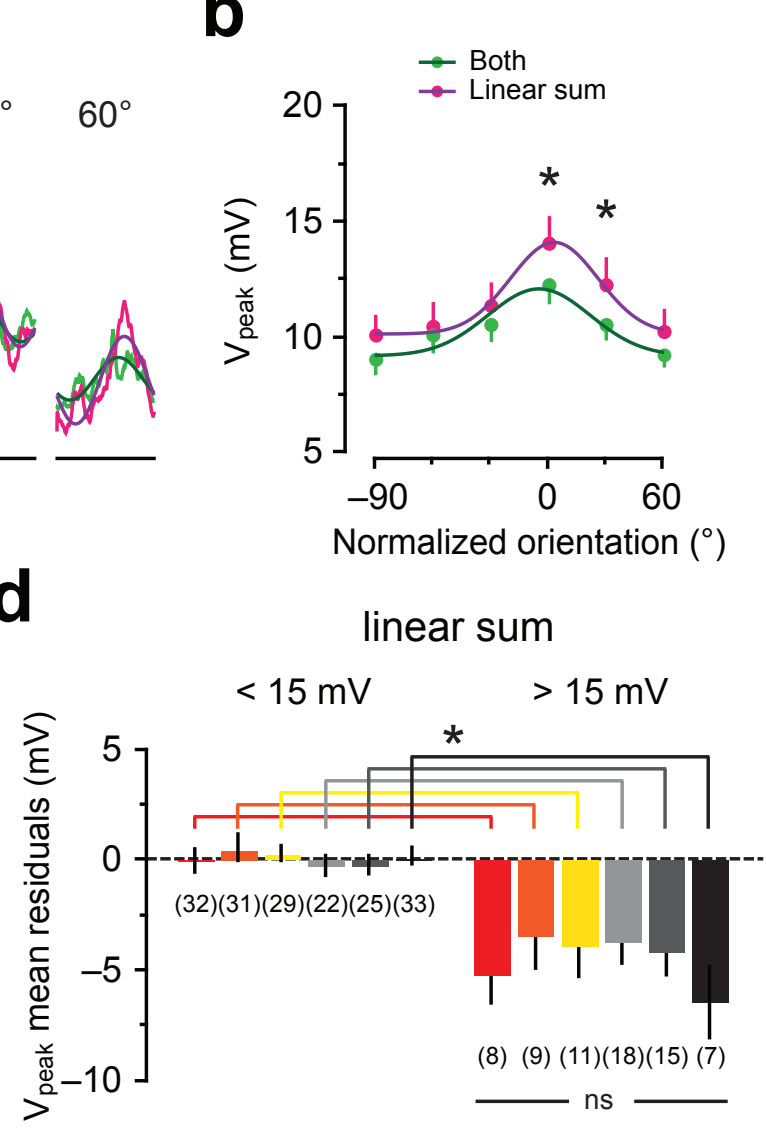

e
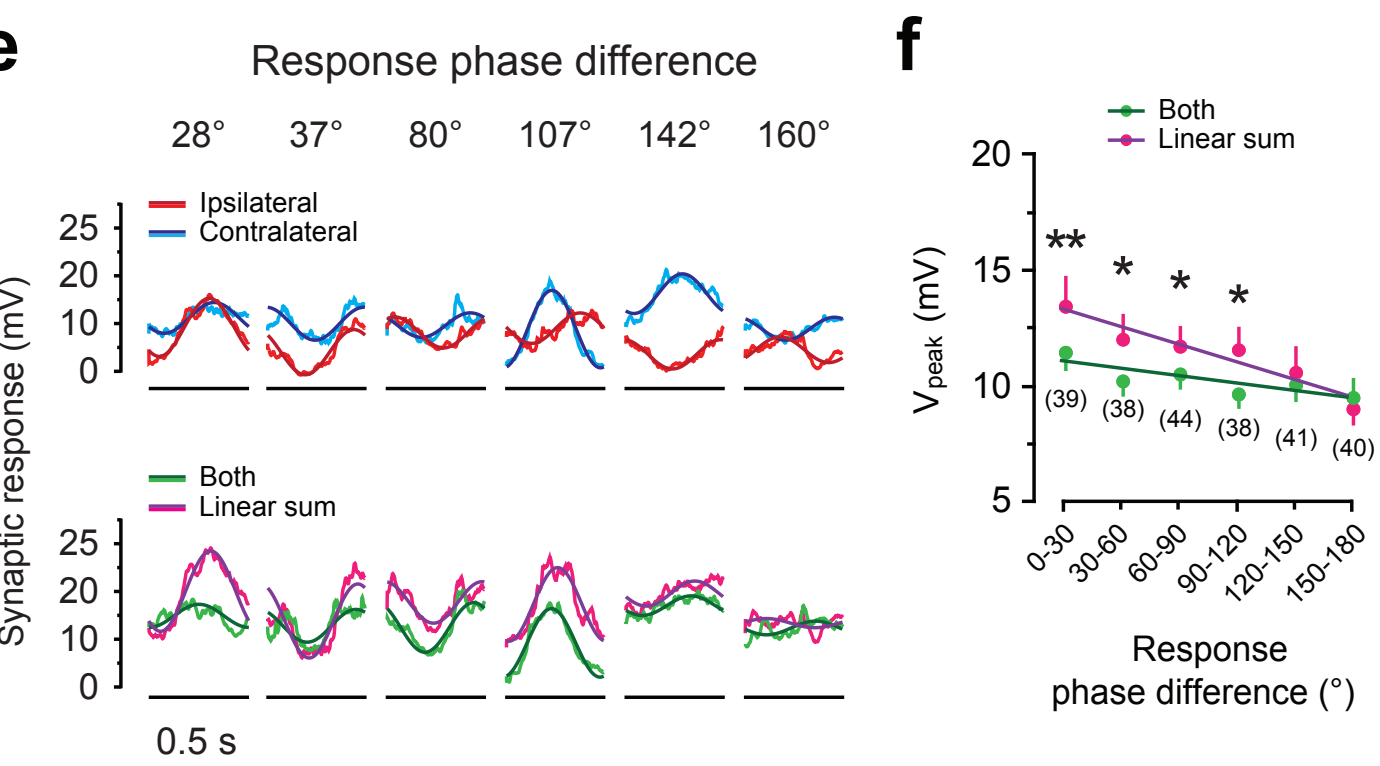

g

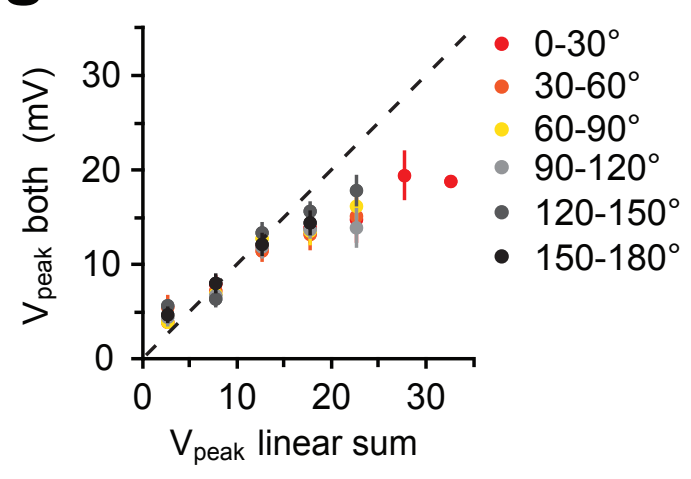

h

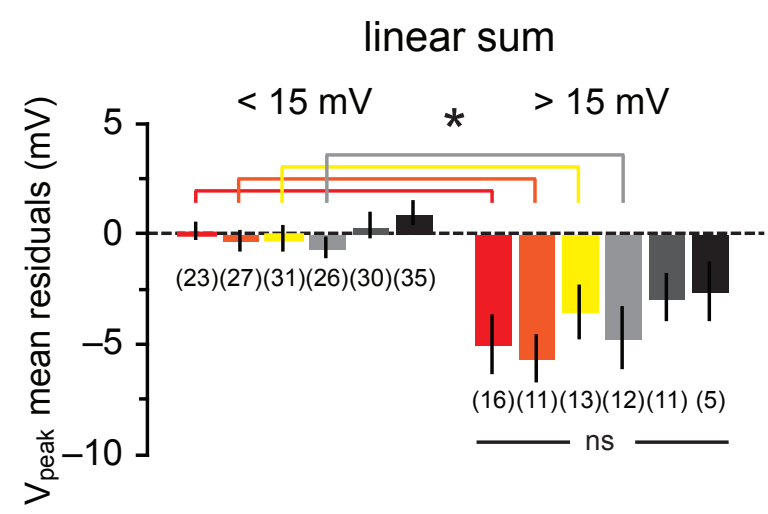


a

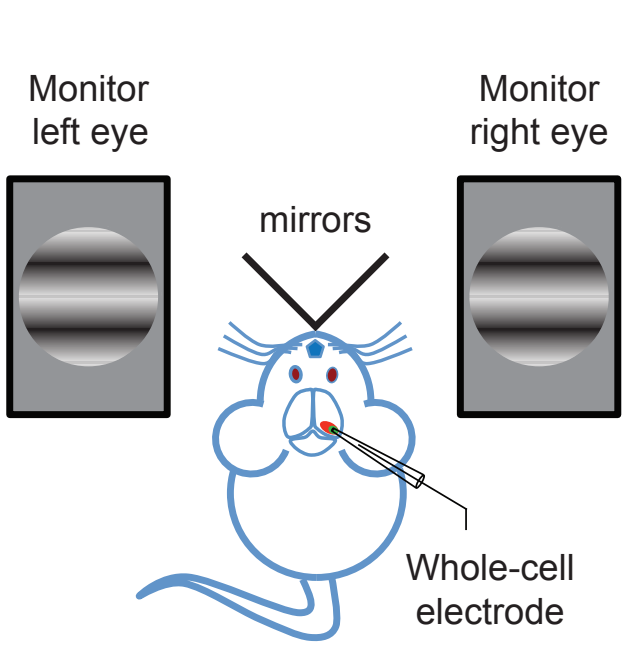

C

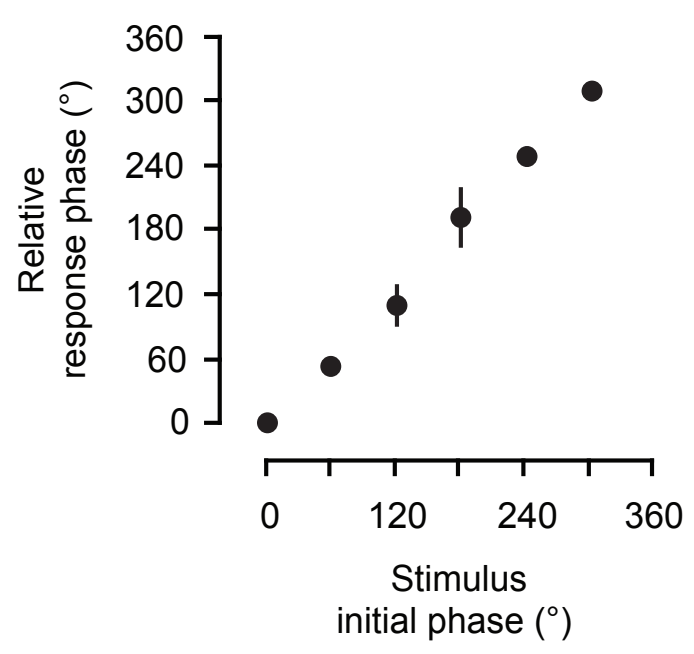

b

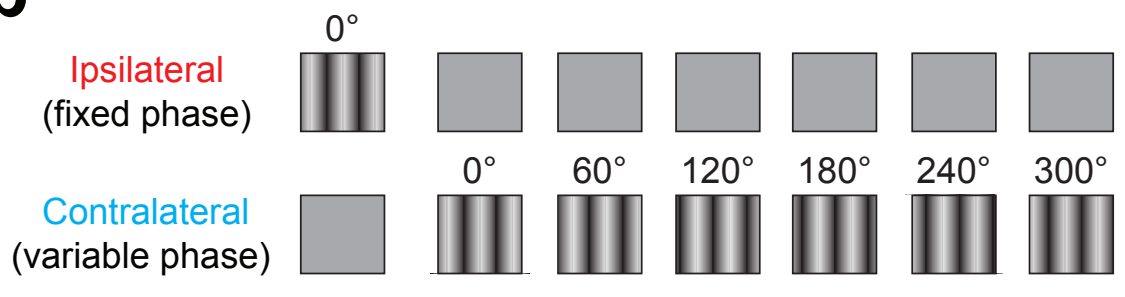

Response phase difference (contralateral - ipsilateral)

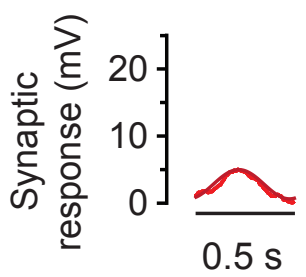

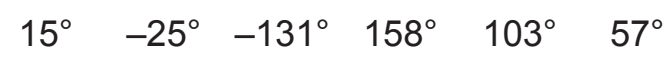

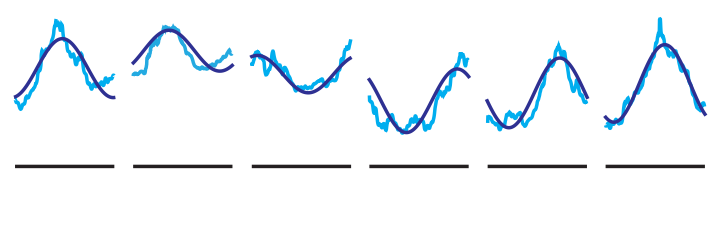

Binocular phase disparity

$0^{\circ} \underset{\text { (preferred) }}{60^{\circ}} 120^{\circ} \quad 180^{\circ} \quad 240^{\circ} \quad 300^{\circ}$

Ipsilateral

(fixed phase)

Contralateral

(variable phase)

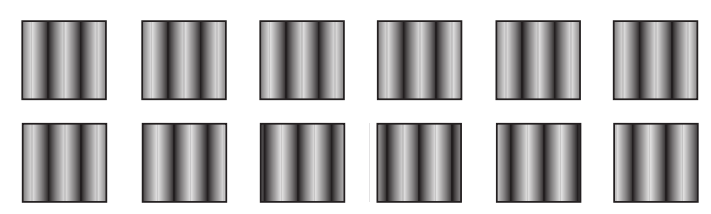

- Both

- Linear sum

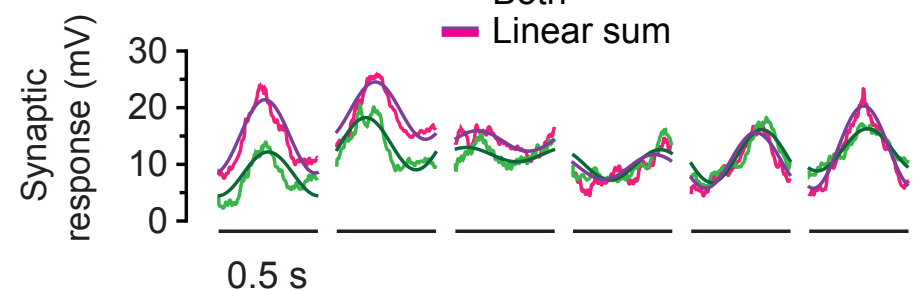

e
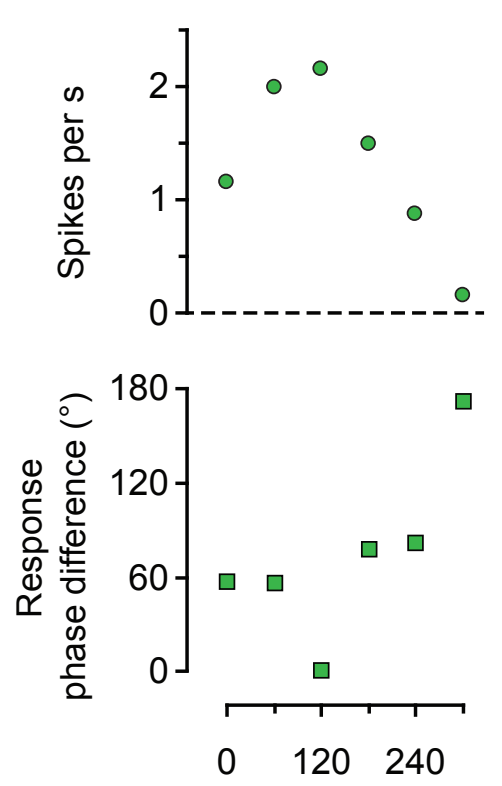

Stimulus phase disparity $\left({ }^{\circ}\right)$ f

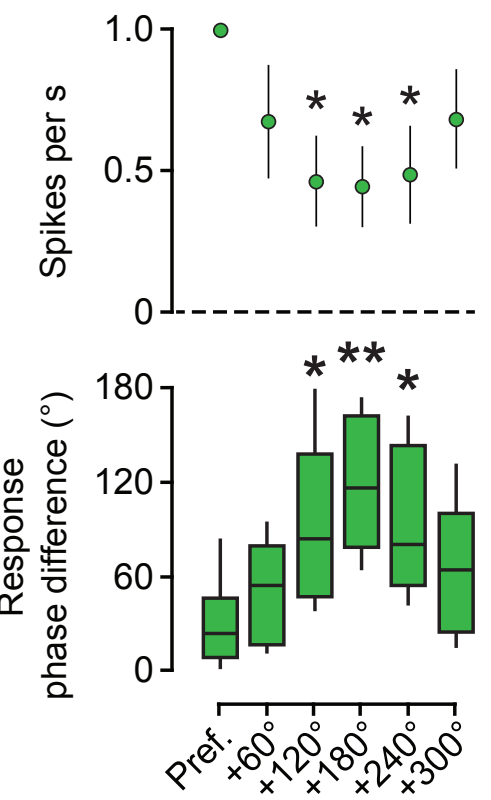

Relative

stimulus phase disparity $\left(^{\circ}\right)$

g

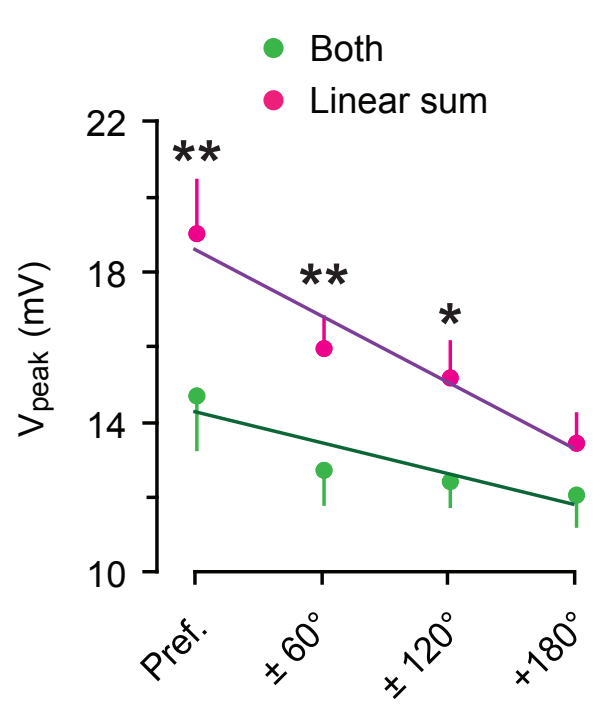

Relative stimuls phase disparity $\left({ }^{\circ}\right)$ 
a

Normalized direction

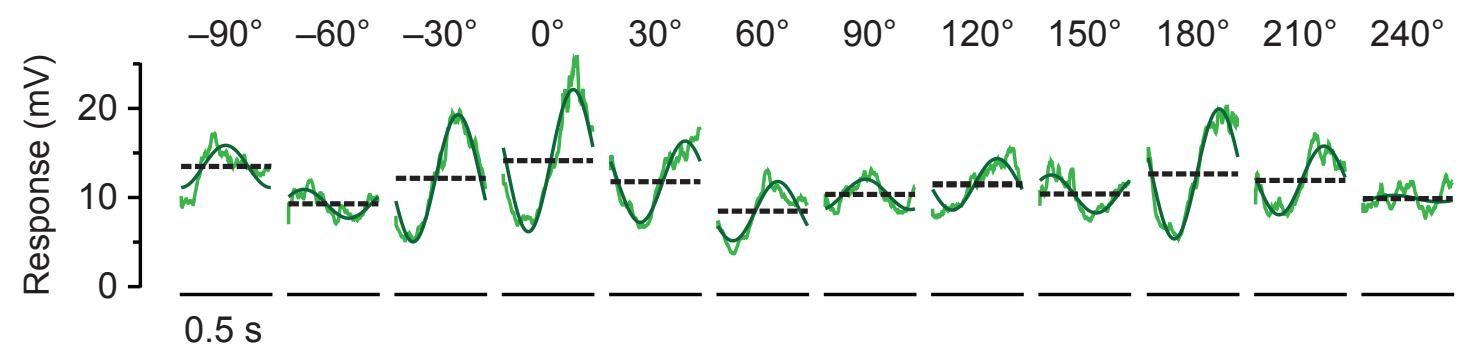

b
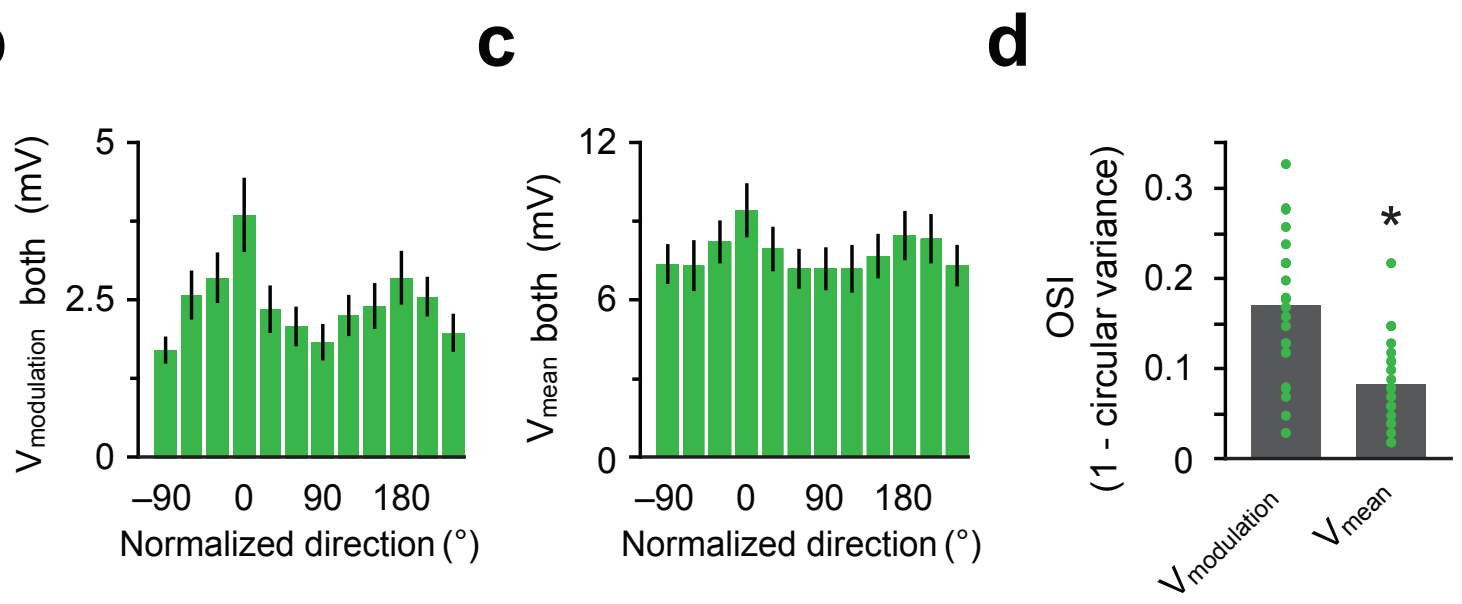

e
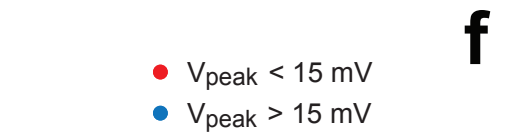

- $\mathrm{V}_{\text {peak }}<15 \mathrm{mV}$

g

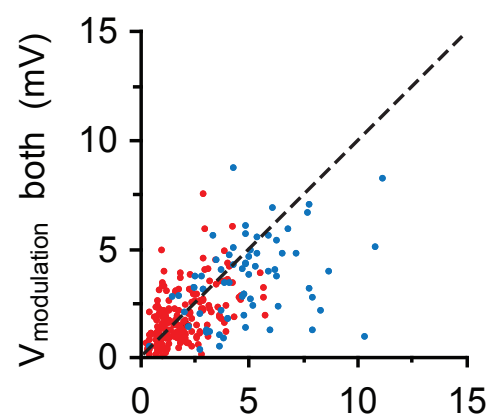

$\mathrm{V}_{\text {modulation }}$ linear sum $(\mathrm{mV})$

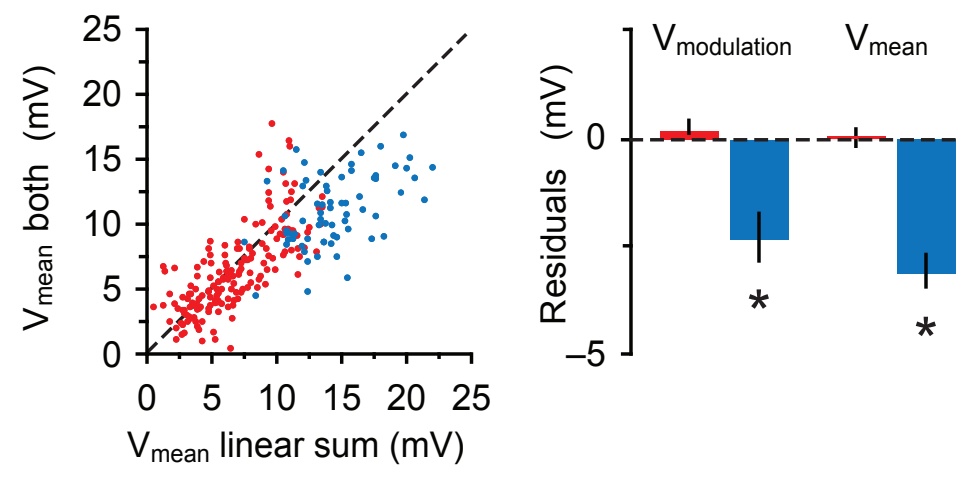

h
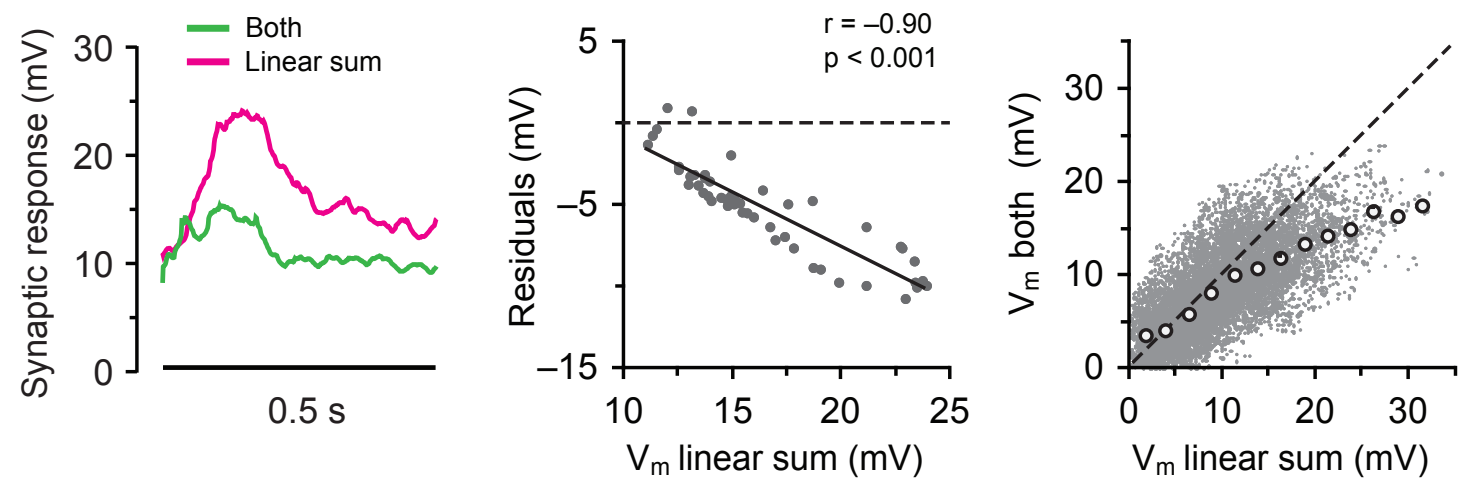
a

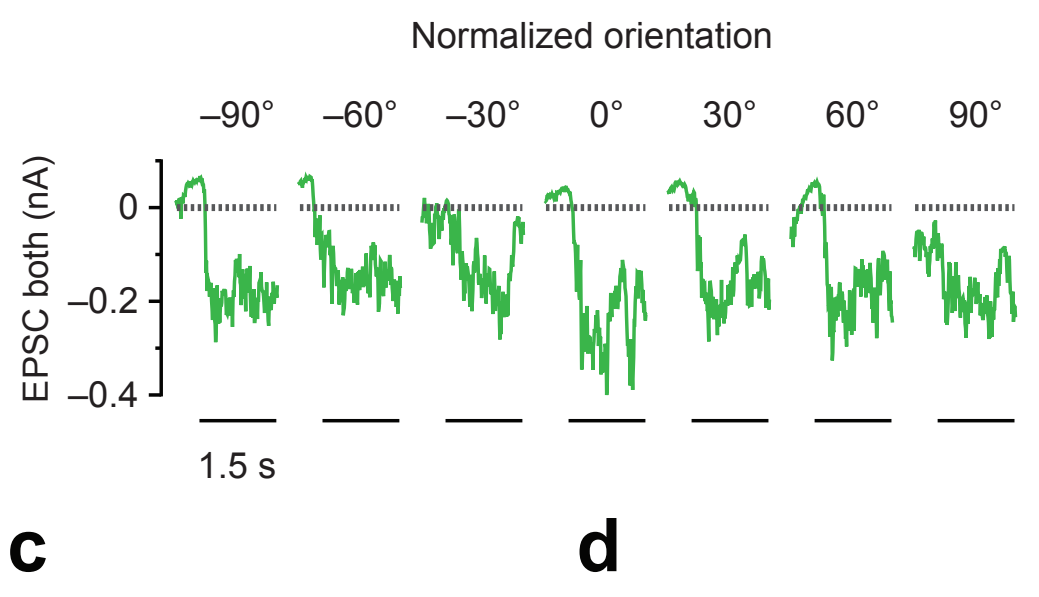

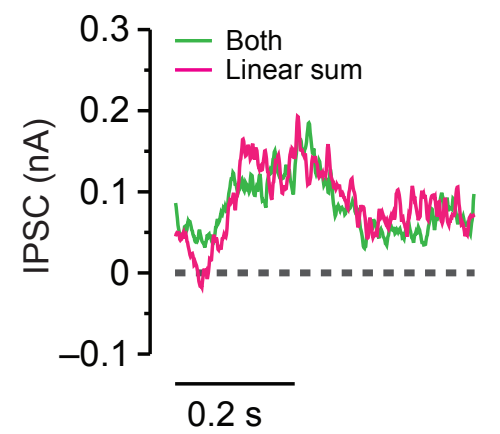
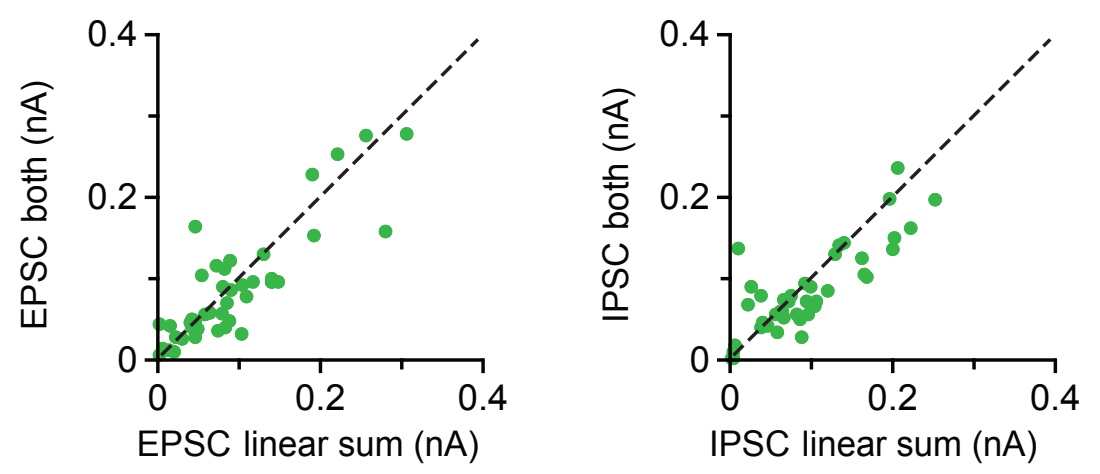

b

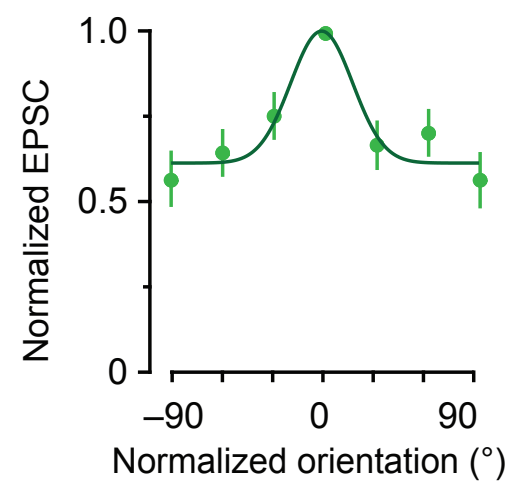

e
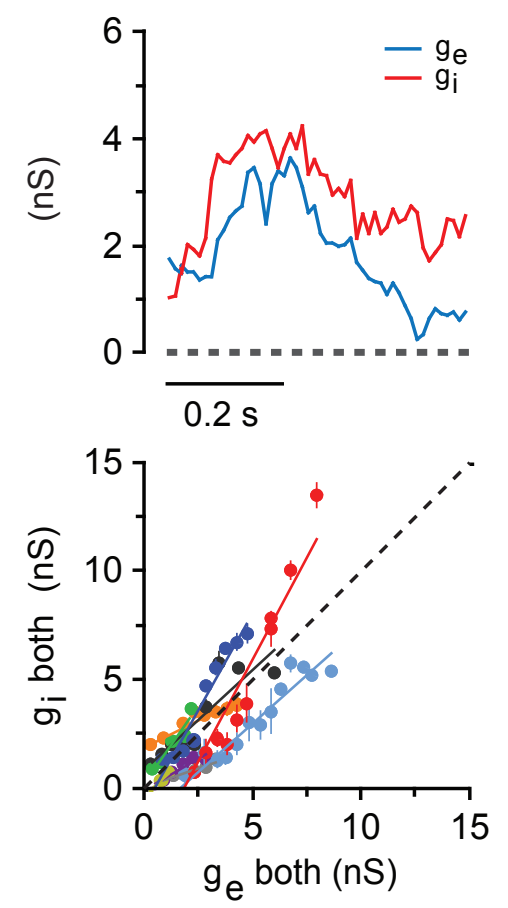
a

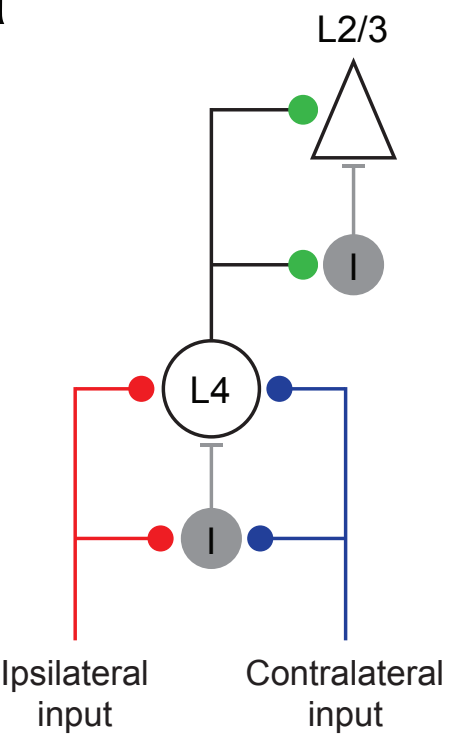

b Common-pool
model

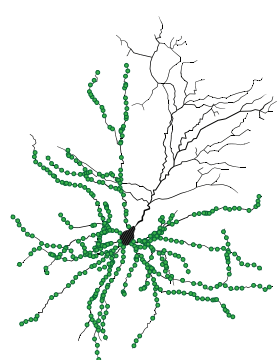

- Excitatory binocular

Dendritic inhibition model

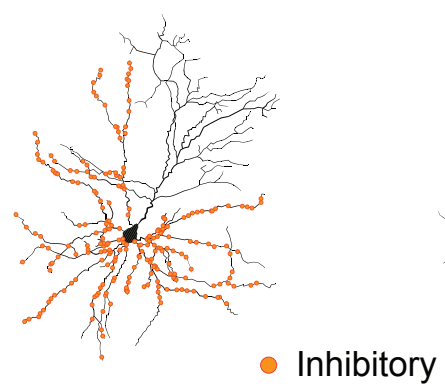

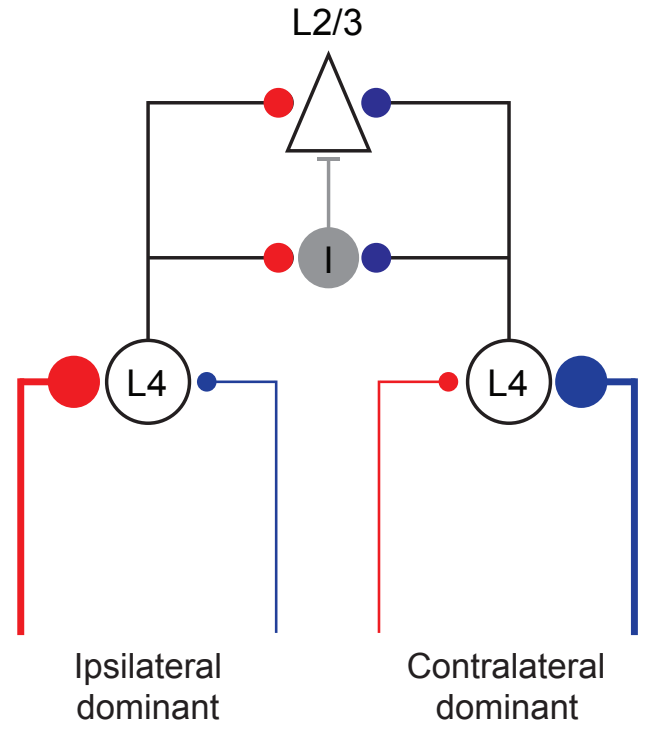

C
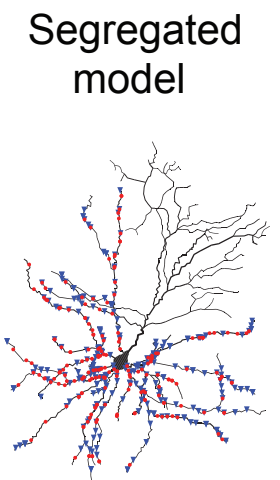

$\checkmark$ Excitatory contralateral

- Excitatory ipsilateral

Somatic inhibition model

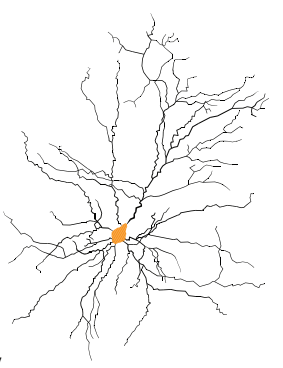

Conductance-based synapses
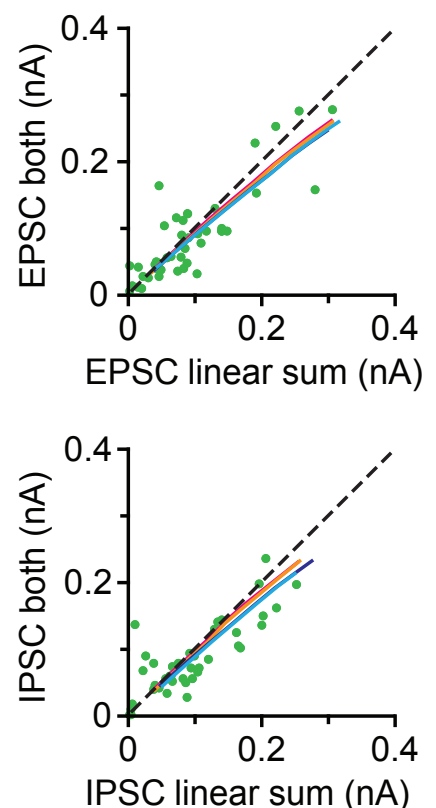

- Common-pool dendritic inhibition

- Common-pool somatic inhibition

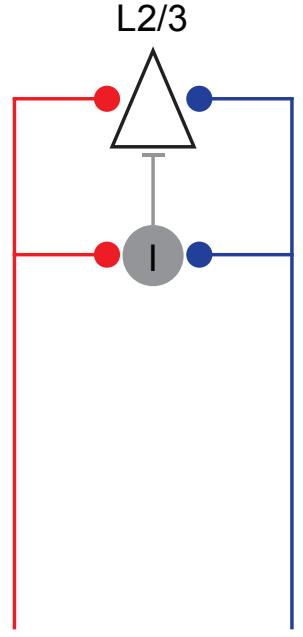

Ipsilateral input

Contralateral input

d

Current-based synapses

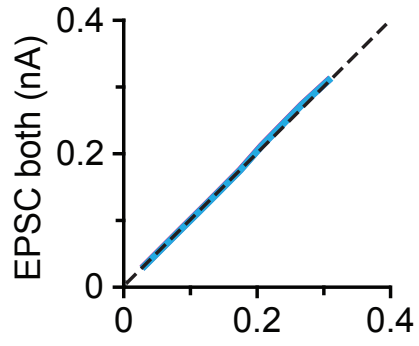

EPSC linear sum (nA)

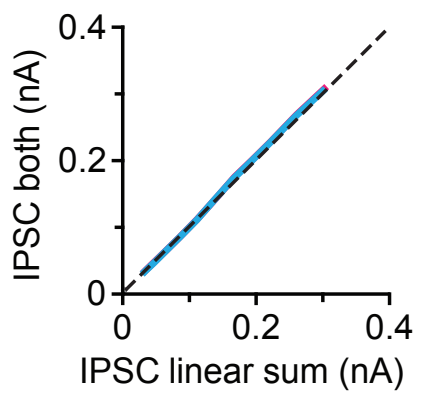

Segregated dendritic inhibition

Segregated somatic inhibition
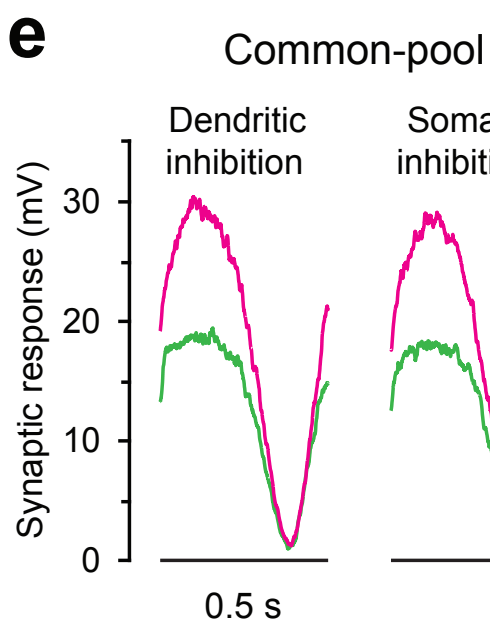

Segregated

Somatic inhibition

Dendritic inhibition

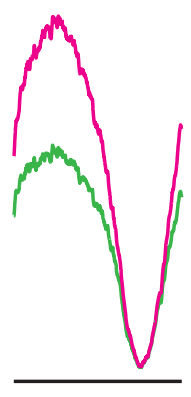

Somatic

inhibition

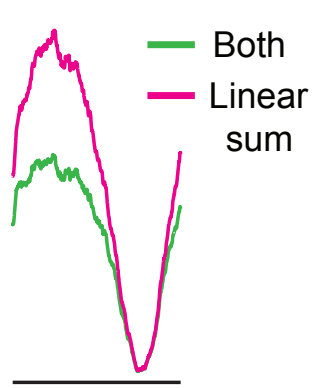

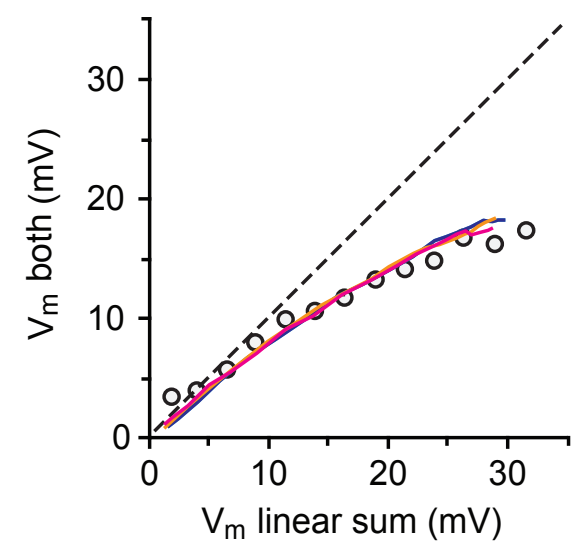


a

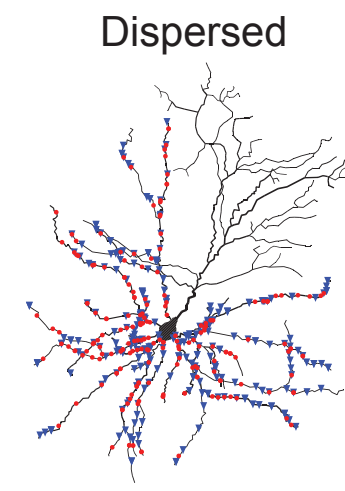

b

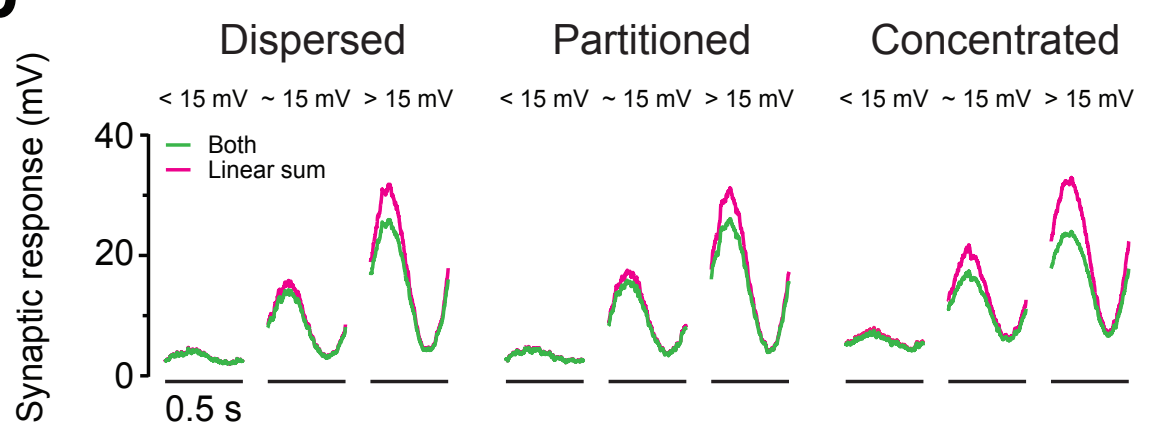

d

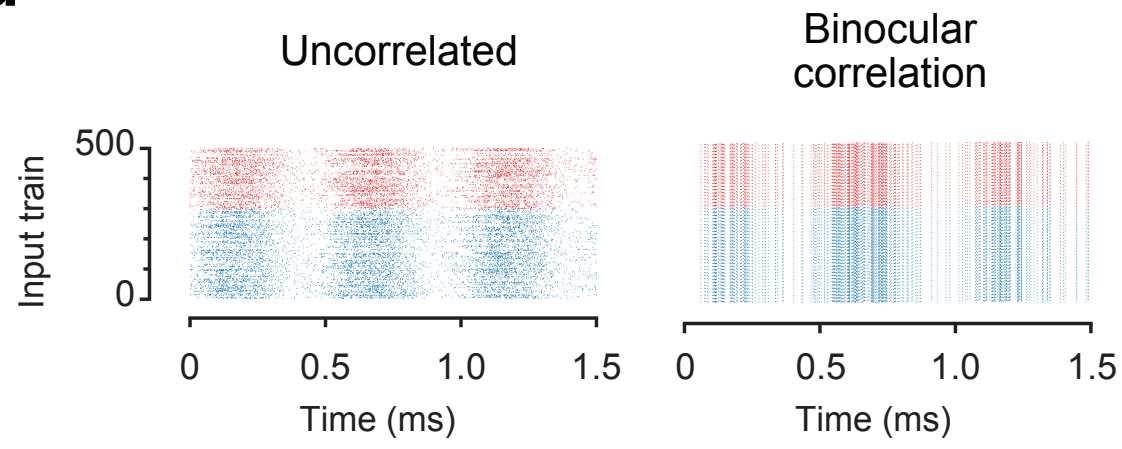

e
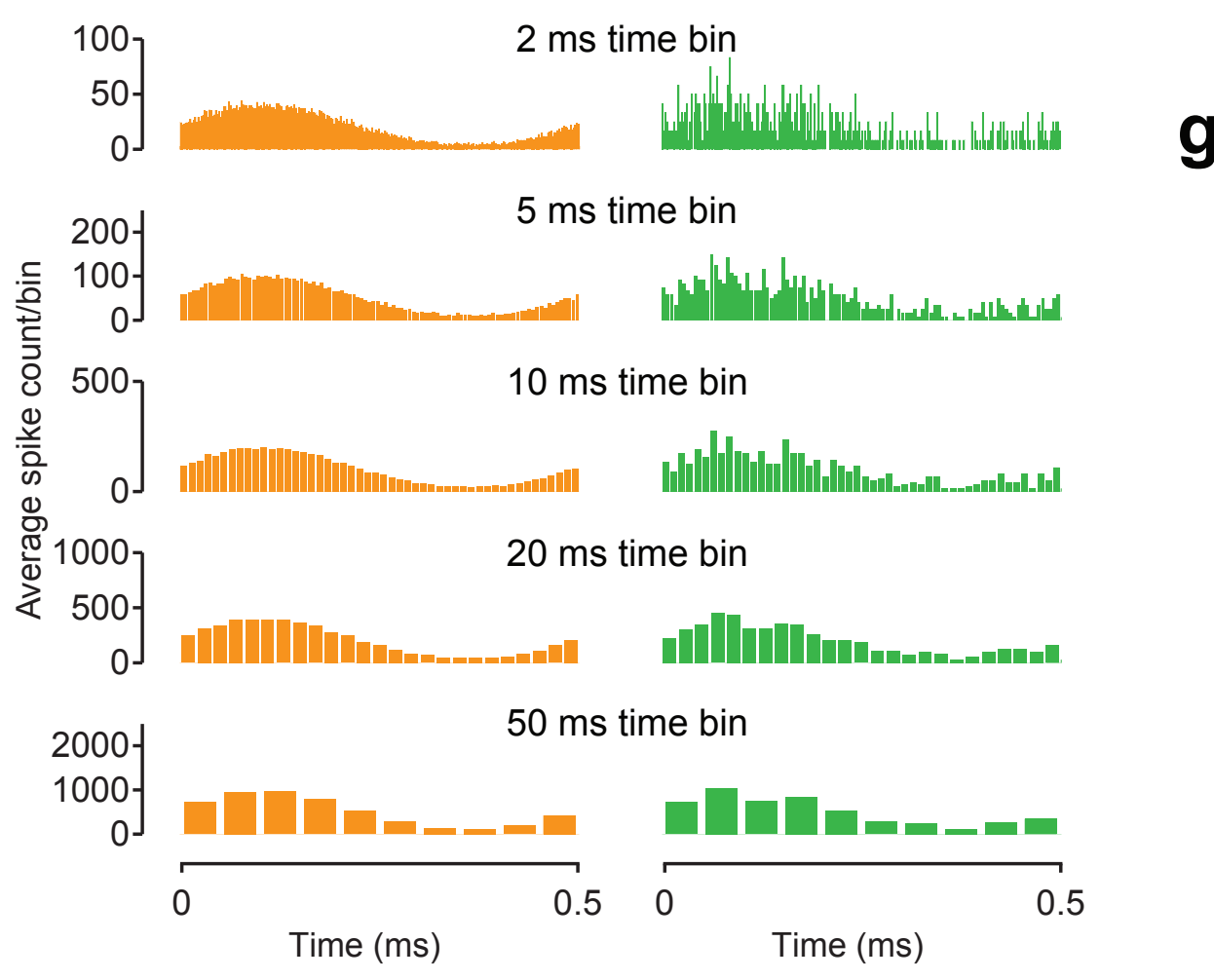

C
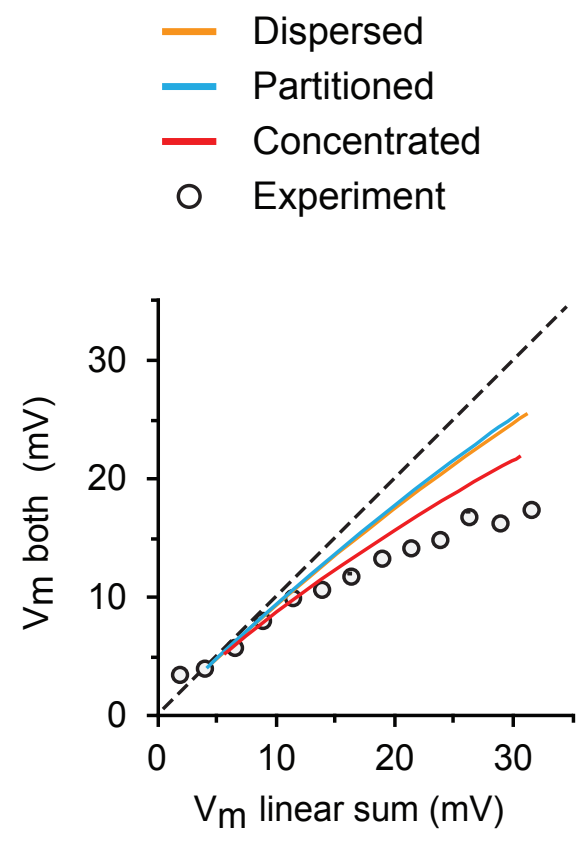

f

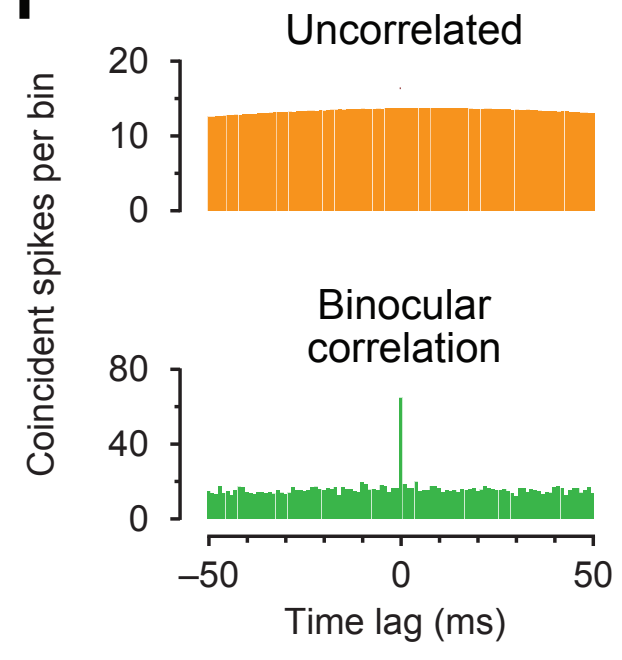

g

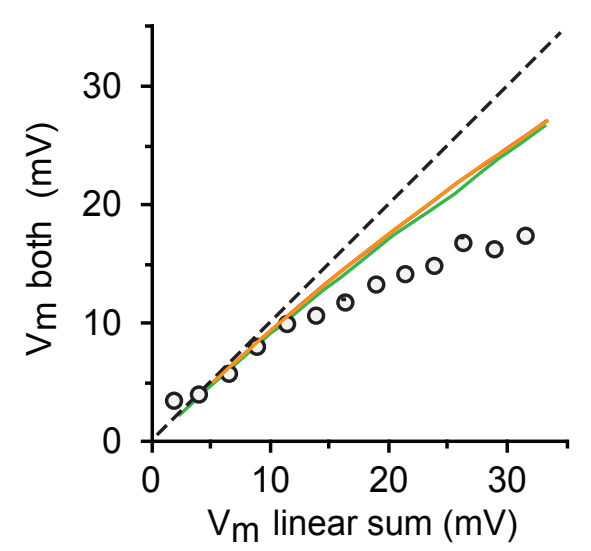


a

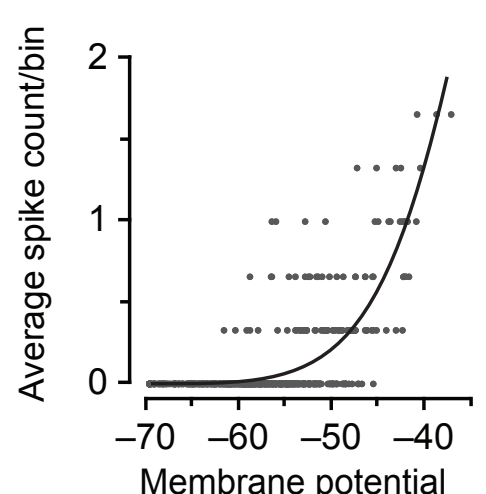

b

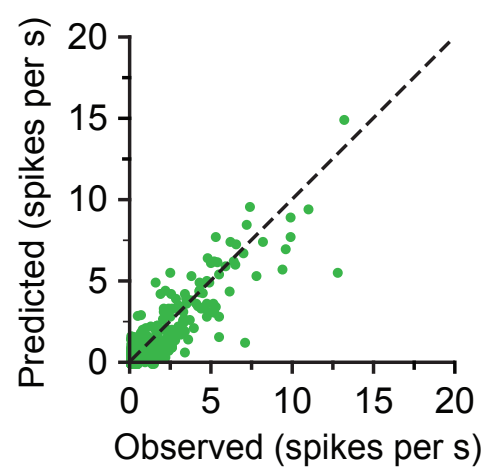

Both
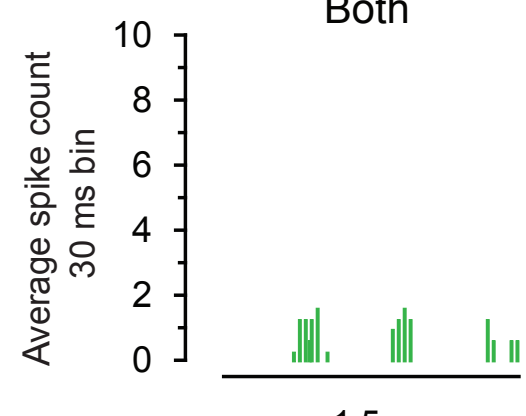

$1.5 \mathrm{~s}$

Both
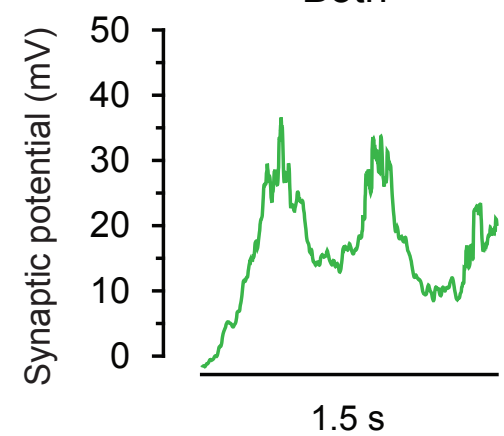

Linear Sum

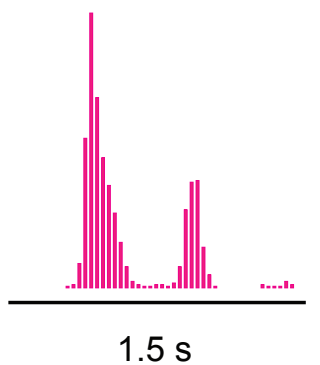

Linear Sum

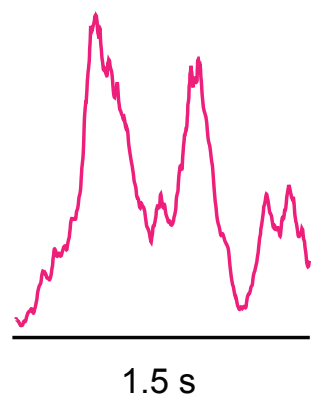

d

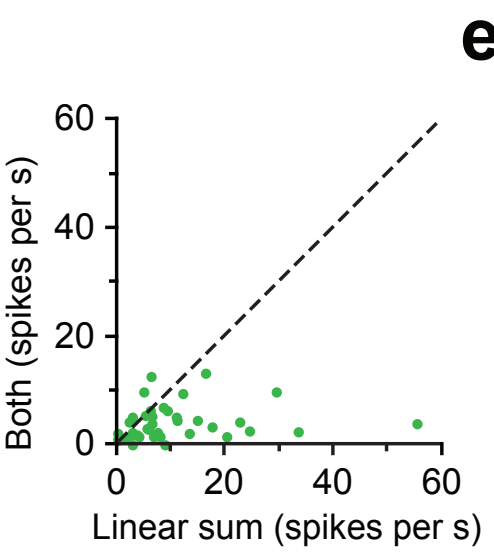

e

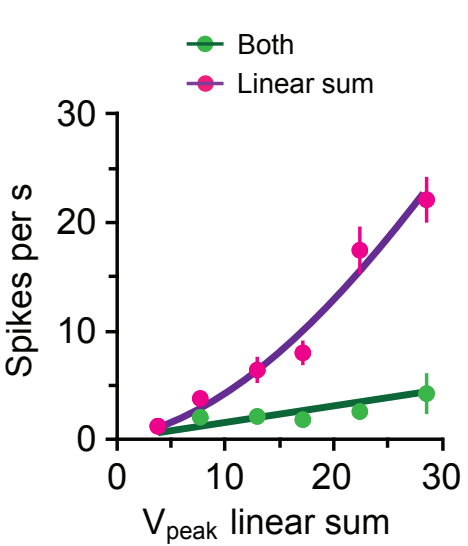

f $\rightarrow$ Both

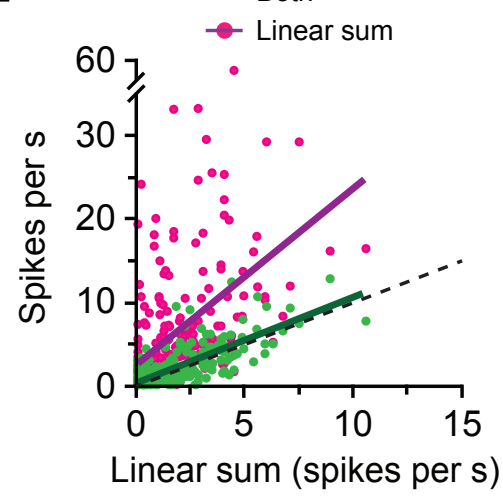

g

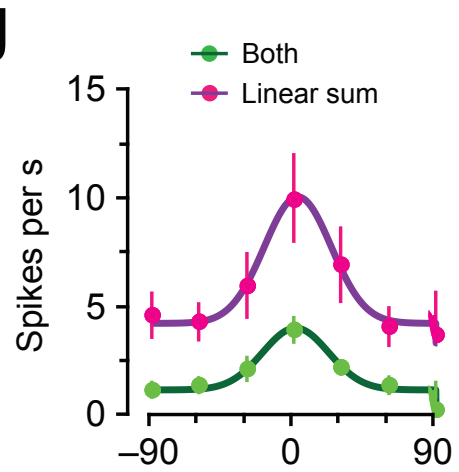

h
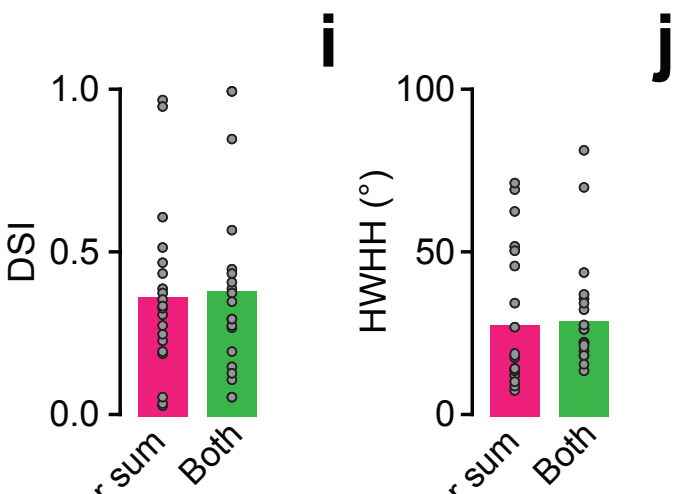

j

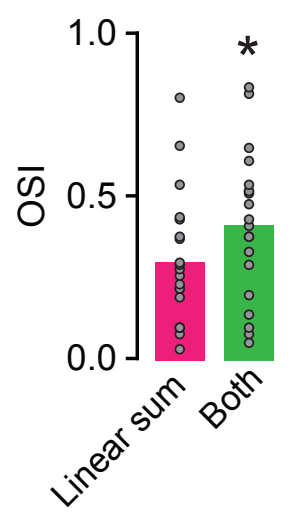

Normalized orientation $\left({ }^{\circ}\right)$ 